\title{
SEMILINEAR POISSON PROBLEMS IN SOBOLEV-BESOV SPACES ON LIPSCHITZ DOMAINS
}

\author{
MARTIN Dindoš* AND MARIUS MitreA ${ }^{\dagger}$
}

\begin{abstract}
Extending recent work for the linear Poisson problem for the Laplacian in the framework of Sobolev-Besov spaces on Lipschitz domains by Jerison and Kenig [16], Fabes, Mendez and Mitrea [9], and Mitrea and Taylor [30], here we take up the task of developing a similar sharp theory for semilinear problems of the type $\Delta u-N(x, u)=F(x)$, equipped with Dirichlet and Neumann boundary conditions.
\end{abstract}

\section{Introduction}

As evidenced by the large body of works (cf., e.g., the monographs $[\mathbf{2 3}],[\mathbf{1 3}],[\mathbf{1 0}],[\mathbf{1 1}],[\mathbf{4}],[\mathbf{1 2}],[\mathbf{3 6}],[\mathbf{1}],[\mathbf{1 4}]$ and the references therein) nonlinear elliptic boundary value problems have become lately the center of considerable attention, especially due to their pivotal role in such diverse disciplines as spectral and scattering theory, differential geometry, mathematical physics, etc. Meanwhile, and particularly more so in the last decade, this field of research has substantially profited from basic progress in many of the related areas in which some of its methods and techniques are rooted.

In $[\mathbf{2 8}],[\mathbf{2 9}],[\mathbf{3 0}],[\mathbf{3 1}],[\mathbf{2 7}]$, the authors have initiated a program aimed at extending the Euclidean, constant coefficient theory from [39], $[\mathbf{6}],[\mathbf{1 6}],[\mathbf{9}]$ to the case of Lipschitz domains in Riemannian manifolds. One notable achievement in [30] is developing a sharp linear theory for the Poisson problem with Dirichlet and Neumann boundary conditions in Lipschitz domains for the Laplace-Beltrami operator, thus extending

2000 Mathematics Subject Classification. Primary: 35J65, 35B65; Secondary: 42B20, $46 \mathrm{E} 35$.

Key words. Nonlinear equations, Lipschitz domains, elliptic PDE's, boundary value problems.

${ }^{*}$ This research was partially conducted by the author for the Clay Mathematics Institute.

${ }^{\dagger}$ Author partly supported by NSF. 
the main results in [16], [9]. The aim of the present paper is to continue this line of work and produce a semilinear version of the aforementioned results, which is in the nature of best possible. A task similar in spirit but in a different functional analytic setting has been accomplished in [7], $[8]$.

In order to be more specific, we momentarily digress for the purpose of introducing some notation and making some definitions. Let $M$ be a smooth, connected, compact, boundaryless manifold, of real dimension $\operatorname{dim} M=n$ (unless explicitly mentioned otherwise, we shall assume that $n \geq 3$ ), equipped with a Riemannian metric tensor $g=$ $\sum_{j, k} g_{j k} d x_{j} \otimes d x_{k}$ whose coefficients satisfy

$$
g_{j k} \in C^{1+\gamma}, \text { some } \gamma>0 .
$$

The Laplace-Beltrami operator on $M$ is then given in local coordinates by

$$
\Delta u:=\operatorname{div}(\operatorname{grad} u)=g^{-1 / 2} \partial_{j}\left(g^{j k} g^{1 / 2} \partial_{k} u\right),
$$

where we use the summation convention, take $\left(g^{j k}\right)$ to be the matrix inverse to $\left(g_{j k}\right)$, and set $g:=\operatorname{det}\left(g_{j k}\right)$. Recall that $\Omega \subset M$ is called a Lipschitz domain provided $\partial \Omega$ can be described in appropriate local coordinates by means of graphs of Lipschitz functions. Also, the Sobolev scale $L_{s}^{p}(M), 1<p<\infty, s \geq 0$, is obtained by lifting $L_{s}^{p}\left(\mathbb{R}^{n}\right):=$ $\left\{(I-\Delta)^{s / 2} f ; f \in L^{p}\left(\mathbb{R}^{n}\right)\right\}$ to $M$. We denote by $L_{s}^{p}(\Omega)$ the restriction of elements in $L_{s}^{p}(M)$ to the Lipschitz domain $\Omega$. As is customary, we set $L_{s, 0}^{p}(\Omega)$ for the subspace consisting of restrictions to $\Omega$ of elements from $L_{s}^{p}(M)$ with support contained in $\bar{\Omega}$. For $s>0$ and $1<p, q<\infty$ with $1 / p+1 / q=1$, we set $L_{-s}^{p}(\Omega):=\left(L_{s, 0}^{q}(\Omega)\right)^{*}$. As is well-known, if $B_{s}^{p, q}(\partial \Omega), 1 \leq p, q \leq \infty, 0<|s|<1$, stands for the usual class of Besov spaces on $\partial \Omega$, then the trace operator $\operatorname{Tr}$ is well-defined from $L_{s}^{p}(\Omega)$ onto $B_{s-1 / p}^{p, p}(\partial \Omega)$ for each $1<p<\infty$ and $1 / p<s<1+1 / p$. For a more detailed exposition, the interested reader is referred to $[\mathbf{3 2}],[\mathbf{3 7}],[\mathbf{3}],[\mathbf{2}]$, and especially [16] for the context of Lipschitz domains.

Returning to the mainstream discussion, here we shall be concerned with the semilinear elliptic PDE

$$
\Delta u-N(x, u)=F(x) \quad \text { in } \quad \Omega \subset M
$$

equipped with either Dirichlet or Neumann boundary conditions. Let us point out that when the nonlinearity $N(x, u)$ is of class $C^{1}$ in $u$ then (1.3) can be rephrased as

$$
\Delta u-a(x, u) u=f \quad \text { in } \quad \Omega,
$$


where

$$
a(x, u):=\int_{0}^{1} \frac{\partial N}{\partial u}(x, t u) d t, \quad \text { and } \quad f(x):=F(x)+N(x, 0) .
$$

Under various growth and smoothness assumptions on $\Omega, N, F$ and $u$, the PDE (1.3) has received a lot of attention in the literature lately. Extending some earlier work in [38], T. Runst and W. Sickel have considered in $[\mathbf{3 2}$, Chapter 6] the case of (1.3) when $\Omega$ is smooth, the nonlinearities are of power type, and $u, F$ belong to Sobolev-Besov-TriebelLizorkin spaces. In [15], V. Isakov and A. I. Nachman have treated (1.3) equipped with a Dirichlet boundary condition in the case of a two dimensional Euclidean Lipschitz domain $\Omega$ and when $u \in L_{1}^{2}(\Omega) \cap C^{0}(\bar{\Omega})$. In [20], J. Johnsen and T. Runst have addressed (1.3) in the framework of Sobolev-Besov-Triebel-Lizorkin spaces in the case of smooth domains and nonlinearities of composition type (i.e., when $N(x, u)=N(u)$ ). Related work can also be found in [18], [13], [5], [22].

In the case of Dirichlet boundary conditions, our main results (cf. Theorem 3.1 and Theorem 3.2) deal with the following situation: $\Omega$ is an arbitrary Lipschitz domain, $u \in L_{s+1 / p}^{p}(\Omega), F \in L_{s+1 / p-2}^{p}(\Omega), 0<s<1$, $1<p<\infty$, and either $N(x, u)$ has sublinear growth in $u$ or $a(x, u)$ (from (1.5)) has an admissible polynomial growth (including a limiting case of exponential behavior). Similar (albeit technically somewhat less refined) results hold in the case of Neumann boundary conditions, even in the presence of (sublinear, power type) nonlinearities in the boundary conditions; cf. Theorem 4.1 for a precise statement.

We are interested in the maximal range of indices $s, p$, for which (1.3) is solvable in the context of Lipschitz domains on the Sobolev-Besov scales. In this respect, we would like to stress that our results are sharp in the sense that they reduce to an optimal linear theory in the absence of nonlinearities. That is, we solve

$$
\begin{aligned}
\Delta u-a(x, u) u & =f \in L_{s+1 / p-2}^{p}(\Omega), \\
\operatorname{Tr} u & =g \in B_{s}^{p, p}(\partial \Omega), \quad u \in L_{s+1 / p}^{p}(\Omega)
\end{aligned}
$$

under admissible growth conditions on $a(x, u)$ (of polynomial and exponential nature) and for the same range of indices $s, p$ as in [30].

The emphasis in the approach we develop is on understanding how the solution of the linear Poisson problem depends on lower order perturbations of the Laplace-Beltrami operator. The main achievement in this regard is to prove that the nonlinear mapping $V \mapsto(\Delta-V)^{-1}$ has 
sublinear growth in $V$. That is, in a suitable functional analytic context,

$$
\begin{aligned}
\left\|(\Delta-V)^{-1}\right\| \leq C(1+\|V\|)^{\theta} & \\
& \text { where } \theta \in[0,1) \text { and } C \text { is independent of } V \geq 0 .
\end{aligned}
$$

See (2.6)-(2.7) for an exact formulation. In turn, this key estimate allows for a convenient implementation of the Schauder fixed-point theorem in the framework of Lebesgue spaces. The proof of (1.7) is a delicate argument which relies on the maximum principle, estimates for fractional powers of the Dirichlet Laplacian and interpolation. In the process, we also show that if $V \in L^{n / 2}$ is nonnegative, then the Schrödinger operator

$$
\Delta-V: L_{s+1 / p, 0}^{p}(\Omega) \longrightarrow L_{s+1 / p-2}^{p}(\Omega)
$$

has the same optimal invertibility range as the unperturbed Laplacian $\Delta$. The integrability exponent $n / 2$ is natural, given the sharp unique continuation results from $[\mathbf{1 7}]$.

The layout of the paper is as follows. In Section 2 we refine the treatment of the linear Dirichlet Poisson problem from [30] by allowing less regular lower order terms and by deriving more precise estimates. This is then used in Section 3 to prove, among other things, the solvability of (1.6). Neumann boundary conditions are considered in Section 4. The case of of nonlinearities $N(x, u)$ with sublinear growth in $u$ is treated in Section 5. This section also contains a more detailed analysis of several relevant examples. Finally, in Section 6, sufficient conditions on the data are produced so that a solution of (1.6) obeying nontangential maximal function estimates can be found. Here we also analyze the case when boundary nonlinearities are allowed.

Acknowledgements. It is a pleasure to thank Michael Taylor for bringing us together and for his constant support and interest in our work. The second named author would also like to thank Winfried Sickel and Jon Johnsen for some helpful conversations.

\section{The linear theory revisited}

We shall retain as much as possible the notation introduced in Section 1. The aim of this section is to prove a refined version of the well-posedness result for the Dirichlet Poisson problem for $\Delta$ on SobolevBesov spaces from $[\mathbf{3 0}]$. 
Theorem 2.1. Let $\Omega \subset M$ be an arbitrary Lipschitz domain and for a function $V$ satisfying

$$
V \geq 0 \quad \text { and } \quad V \in L^{r}(M) \text { for some } r \geq n / 2,
$$

set $L:=\Delta-V$. Then there exists $\varepsilon=\varepsilon(\Omega)>0$ with the following significance. Let $0<s<1$ and $1<p<\infty$ satisfy at least one of the following conditions:

$$
\begin{array}{lll}
\frac{2}{1+\varepsilon}<p<\frac{2}{1-\varepsilon} & \text { and } & 0<s<1 \\
1 \leq p<\frac{2}{1+\varepsilon} & \text { and } & \frac{2}{p}-1-\varepsilon<s<1 \\
\frac{2}{1-\varepsilon}<p \leq \infty & \text { and } & 0<s<\frac{2}{p}+\varepsilon,
\end{array}
$$

and, in addition,

$$
\frac{1}{r}-\frac{2}{n}<\frac{n-1}{n p}-\frac{s}{n}<1-\frac{1}{r} .
$$

Then the Dirichlet Poisson problem

$$
(D P)\left\{\begin{array}{l}
L u=f \in L_{s+\frac{1}{p}-2}^{p}(\Omega), \\
\operatorname{Tr} u=g \in B_{s}^{p, p}(\partial \Omega), \\
u \in L_{s+\frac{1}{p}}^{p}(\Omega),
\end{array}\right.
$$

has a unique solution, which also satisfies

$$
\|u\|_{L_{s+\frac{1}{p}}^{p}(\Omega)} \leq C\left(\|f\|_{L_{s+\frac{1}{p}-2}^{p}(\Omega)}+\|g\|_{B_{s}^{p, p}(\partial \Omega)}\right)
$$

for some $C=C(\Omega, V, p, s)>0$.

Introduce $\tau:=n p /(n-1-s p)$ if $s p<n-1$ and $\tau:=\infty$ if $s p>n-1$. Then, if $1 / p \leq s$ and $s p \neq n-1$, there exists a constant $C=C(\Omega, p, s)>$ 0 , independent of $V$, such that

$$
\|u\|_{L^{\tau}(\Omega)} \leq C\left(\|f\|_{L_{s+\frac{1}{p}-2}^{p}(\Omega)}+\|g\|_{B_{s}^{p, p}(\partial \Omega)}\right) .
$$


If $s p=n-1$ then for some $C>0$ independent of $V$ and any $\tau \in[2, \infty)$,

$$
\|u\|_{L^{\tau}(\Omega)} \leq C \tau^{1-1 / p}\left(\|f\|_{L_{s+\frac{1}{p}-2}^{p}(\Omega)}+\|g\|_{B_{s}^{p, p}(\partial \Omega)}\right) .
$$

On the other hand, if $s<1 / p$, then the estimate

$$
\|u\|_{L^{\tau}(\Omega)} \leq C\left(1+\|V\|_{L^{r}(\Omega)}\right)^{\theta}\left(\|f\|_{L_{s+\frac{1}{p}-2}^{p}(\Omega)}+\|g\|_{B_{s}^{p, p}(\partial \Omega)}\right),
$$

holds with

$$
\theta:= \begin{cases}\varepsilon+\frac{r}{2(r-1)}\left(\frac{1}{p}-s\right), & \text { if } r \geq 1+(1-s p) /(2 p-2) \\ \varepsilon+\frac{1}{2-n / r}((n-1) / p-s-n+2), & \text { if } r<1+(1-s p) /(2 p-2)\end{cases}
$$

where $\varepsilon>0$ is arbitrarily small and $C=C(\Omega, s, p, \varepsilon)>0$ is independent of $V$.

In dimension $n=2$, results similar in spirit hold provided (2.2) is replaced by

$$
\begin{array}{lll}
\frac{1}{2}-\varepsilon<\frac{1}{p}<\frac{1}{2}+\varepsilon & \text { and } & 0<s<1 \\
\frac{1}{2}+\varepsilon \leq \frac{1}{p}<1 & \text { and } & \frac{1}{p}-\frac{1}{2}-\varepsilon<s<1 \\
0<\frac{1}{p} \leq \frac{1}{2}-\varepsilon & \text { and } & 0<s<\frac{1}{p}+\frac{1}{2}+\varepsilon
\end{array}
$$

where $\varepsilon=\varepsilon(\partial \Omega) \in\left(0, \frac{1}{2}\right]$. More specifically, the estimates $(2.5)-(2.7)$ hold unchanged, whereas (2.8) holds if also $r>4 / 3$.

Finally, when $\partial \Omega \in C^{1}$, instead of (2.2) when $n \geq 3$, or (2.10) when $n=2$, we may simply allow $s \in(0,1), p \in(1, \infty)$.

Parenthetically, we note that when $r>n$ then (2.3) is satisfied for any $s \in(0,1), p \in(1, \infty)$. Another simple yet useful remark is that, if $\varepsilon>0$ is small enough, then

(2.11) $n \geq 3, r>\frac{n}{2}, s, p$ as in (2.2) with $\varepsilon>0$ small $\Longrightarrow(2.3)$ holds.

Furthermore, in the two dimensional context,

(2.12) $\quad r>\frac{4}{3}, s, p$ as in $(2.10)$

with $\varepsilon>0$ small $\Longrightarrow(2.3)$ holds automatically. 


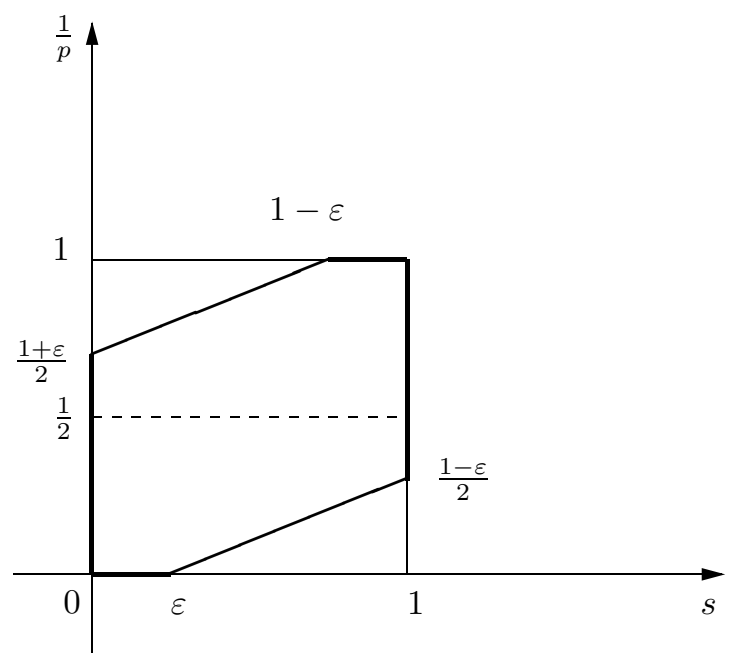

FiguRE 1. The hexagon describing the well posedness region $(2.2)$.

Before presenting the proof of the Theorem 2.1, we need to discuss several auxiliary results.

Lemma 2.2. Let $\Omega$ be a Lipschitz domain (of dimension $\geq 2$ ) and fix $1<p<\infty, 0<s<1, n / 2 \leq r \leq \infty$, so that (2.3) holds. Then the inclusion

$$
L^{r}(\Omega) \cdot L_{s+1 / p}^{p}(\Omega) \hookrightarrow L_{s+1 / p-2}^{p}(\Omega)
$$

is compact.

Proof: Consider first the case when $s p<n-1$ and $r>n / 2$. In this scenario, we shall show that there exists $\varepsilon=\varepsilon(n, p, s, r)>0$ such that

$$
L^{r}(\Omega) \cdot L_{s+1 / p}^{p}(\Omega) \hookrightarrow L_{s+\varepsilon+1 / p-2}^{p}(\Omega) .
$$

In concert with Rellich's selection lemma, this proves that, under the current assumptions, multiplication by an element from $L^{r}(\Omega)$ is a compact operator from $L_{s+1 / p}^{p}(\Omega)$ into $L_{s+1 / p-2}^{p}(\Omega)$.

To see $(2.14)$, if $1 / p_{0}:=1 / p-(s+1 / p) / n$ it follows that $1<p_{0}<\infty$ and $L_{s+1 / p}^{p}(\Omega) \hookrightarrow L^{p_{0}}(\Omega)$. Going further, if $1 / p_{1}:=1 / r+1 / p_{0}$ then $1<p_{1}<\infty$, thanks to $(2.3)$, and we have $L^{r}(\Omega) \cdot L^{p_{0}}(\Omega) \hookrightarrow L^{p_{1}}(\Omega)$. Now, since $L_{s+\varepsilon+1 / p-2}^{p}(\Omega)=\left(L_{2-s-\varepsilon-1 / p, 0}^{p^{\prime}}(\Omega)\right)^{*}$ with $1 / p+1 / p^{\prime}=1$, it 
suffices to prove that $L_{2-s-\varepsilon-1 / p}^{p^{\prime}}(\Omega) \hookrightarrow L^{p_{2}}(\Omega)$, where $1 / p_{2}+1 / p_{1}=1$. This, however, is guaranteed by the estimate $1 / p_{2}>1 / p^{\prime}-(2-s-1 / p) / n$. Some simple algebra now shows that this is always the case provided $r>n / 2$. When $s p \geq n-1, r>n / 2$, one proceeds analogously, choosing $p_{0} \nearrow \infty$.

Finally, when $r=n / 2$, the well-definiteness of the inclusion (2.13) is proved similarly. Then, based on this, (2.14) and an approximation argument, it follows that (2.13) is compact as well.

Lemma 2.3. Let $\Omega$ be a Lipschitz domain and assume that $s, p$ satisfy at least one of the conditions in (2.2). Also, fix a nonnegative function $V \in L^{r}(M)$, with $r \geq n / 2$, and suppose that (2.3) holds. Then

$$
u \in L_{s+1 / p}^{p}(\Omega),(\Delta-V) u \leq 0, \operatorname{Tr} u \geq 0 \Longrightarrow u \geq 0 .
$$

In particular, if $V_{j} \in L^{r}(M), r \geq n / 2$, are such that $0 \leq V_{1} \leq V_{2}$, then

$$
\begin{aligned}
u_{j} \in L_{s+1 / p}^{p}(\Omega), \operatorname{Tr} u_{1} \geq \operatorname{Tr} u_{2} & \geq 0, \\
\left(\Delta-V_{1}\right) u_{1} & \leq\left(\Delta-V_{2}\right) u_{2} \leq 0 \Longrightarrow u_{1} \geq u_{2} .
\end{aligned}
$$

Analogous results are valid for $n=2$, provided that $s, p$ satisfy at least one of the conditions in (2.10). Finally, when $\partial \Omega \in C^{1}$, any $s \in(0,1)$, $p \in(1, \infty)$ will do (in all dimensions) as long as $(2.3)$ is satisfied.

Proof: For an arbitrary, fixed $u \in L_{s+1 / p}^{p}(\Omega)$, let us set $f:=(\Delta-V) u \in$ $L_{s+1 / p-2}^{p}(\Omega)$ and $g:=\operatorname{Tr} u \in B_{s}^{p, p}(\partial \Omega)$. Thus, by assumption, $f \leq 0$ and $g \geq 0$.

For starters, we make the claim that there exist $f_{j} \in C_{\text {comp }}^{\infty}(\Omega), g_{j} \in$ $\operatorname{Lip}(\partial \Omega)$, such that $f_{j} \leq 0, g_{j} \geq 0$, and $f_{j} \rightarrow f$ in $L_{s+1 / p-2}^{p}(\Omega), g_{j} \rightarrow g$ in $B_{s}^{p, p}(\partial \Omega)$. Indeed, the approximating sequence $\left\{g_{j}\right\}_{j}$ can be produced via a standard localization and mollifying procedure. There remains to prove that $f$ belongs to the closure of the convex set $\mathcal{C}:=\{\psi \in$ $\left.C_{\text {comp }}^{\infty}(\Omega) ; \psi \leq 0\right\}$ in $L_{s+1 / p-2}^{p}(\Omega)$. Assuming the opposite (and seeking a contradiction), the Hahn-Banach theorem ensures the existence of some $\Phi \in\left(L_{s+1 / p-2}^{p}(\Omega)\right)^{*}=L_{2-s-1 / p, 0}^{p^{\prime}}(\Omega), 1 / p+1 / p^{\prime}=1$, such that $\langle\Phi, \phi\rangle \leq$ $0<\langle\Phi, f\rangle$ for each $\phi \in \mathcal{C}$. (Strictly speaking, what the Hahn-Banach theorem originally gives is the previous double inequality with 'zero' replaced by some real number $\lambda$. However, given that $\mathcal{C}$ is in fact a cone, it is easy to see that we can always assume that $\lambda=0$.) Now, the first inequality implies $\Phi \geq 0$ which, in turn, contradicts the second, given that $f \leq 0$. This finishes the proof of the claim made at the beginning of the paragraph. 
Next, granted the current assumptions, we will show in the course of the proof of Theorem 2.1 that $u$ (is uniquely determined by, and) depends continuously on $f, g$. Consequently, thanks to a limiting argument (whose implementation is routine, given the claim above), it suffices to prove (2.15) in the case when $f \in L^{\infty}(\Omega)$ and $g \in C^{0}(\partial \Omega)$, which we will assume hereafter. It is not difficult to see that these extra assumptions entail $u \in C^{0}(\bar{\Omega}) \cap C_{\mathrm{loc}}^{1}(\Omega)$.

With an eye on (2.15) and seeking a contradiction, assume next that there exists $x_{0} \in \Omega$ such that $u\left(x_{0}\right)<0$. If we let $\mathcal{O}$ be the connected component of the set $\{x \in \Omega ; u(x)<0\}$ which contains $x_{0}$ and recall that $\left.u\right|_{\partial \Omega}>0$, it follows that $\mathcal{O} \subset \subset \Omega$.

Let $\psi \in C^{\infty}(\mathbb{R})$ be a Lipschitz, non-decreasing, odd function such that $\psi(t)=0$ for $|t| \leq 1$ and $\psi(t)=t$ for $|t| \geq 2$. Set $u_{i}(x):=i^{-1} \psi(i u(x))$, $x \in \overline{\mathcal{O}}, i=1,2, \ldots$. Then, so we claim,

$$
\begin{aligned}
u_{i} \in C_{\text {comp }}^{1}(\mathcal{O}), u_{i} \leq 0 & \\
& \text { and }\left\|u-u_{i}\right\|_{L^{\infty}(\mathcal{O})},\left\|\nabla u-\nabla u_{i}\right\|_{L^{2}(\mathcal{O})} \longrightarrow 0 .
\end{aligned}
$$

Indeed, that $u_{i}$ is compactly supported in $\mathcal{O}$ and $\left\|\nabla u-\nabla u_{i}\right\|_{L^{2}(\mathcal{O})} \rightarrow 0$ are consequences of $\left.u\right|_{\partial \mathcal{O}}=0$ and definitions. As for $\left\|\nabla u-\nabla u_{i}\right\|_{L^{2}(\mathcal{O})} \rightarrow$ 0 , note that $\nabla u_{i}(x)=\psi^{\prime}(i u(x)) \nabla u(x) \rightarrow \nabla u(x)$ as $i \rightarrow \infty$, for each $x \in \mathcal{O}$. Since $\psi$ is Lipschitz and $u \in C^{1}(\overline{\mathcal{O}})$, Lebesgue's dominated convergence theorem then yields the desired conclusion. Going further,

$$
\begin{aligned}
\iint_{\mathcal{O}}|\nabla u|^{2}+V|u|^{2} & =\lim \iint_{\mathcal{O}}\left\langle\nabla u, \nabla u_{i}\right\rangle+V u u_{i} \\
& =-\lim \iint_{\mathcal{O}} u_{i}(\Delta-V) u \leq 0,
\end{aligned}
$$

which contradicts the fact that $u$ is not identically zero in $\mathcal{O}$. This finishes the proof of the implication (2.15).

Finally, to justify (2.16), it suffices to apply (2.15) to the difference $u:=u_{1}-u_{2}$ (with the choice $\left.V:=V_{1}\right)$.

Lemma 2.4. For each Lipschitz domain $\Omega \subset M$ and $1<p<\infty$ there exists a constant $C=C(M, \Omega, p)>0$ such that for any $u \in L_{n / p}^{p}(\Omega)$ and $\tau \in[2, \infty)$

$$
\|u\|_{L^{\tau}(\Omega)} \leq C \tau^{1-1 / p}\|u\|_{L_{n / p}^{p}(\Omega)} .
$$

Furthermore, if $n \geq 3$ and $n-1<p<\infty$, then

$$
\|f\|_{L_{2 / \tau, 0}^{\tau}(\Omega)} \leq C \tau^{1-1 / p}\|f\|_{L_{n / p, 0}^{p}(\Omega)}, \quad \text { for all } \tau \in[p, \infty),
$$

where $C=C(M, \Omega, n, p)>0$ is independent of $\tau$. 
Proof: Clearly, it suffices to deal with the Euclidean version of (2.19). For $p=2$ the statement can be found in [36, Chapter 13] and is a key ingredient in the proof of Trudinger's inequality. The argument used there readily extends, so we only outline the main steps. If $u \in L_{n / p}^{p}\left(\mathbb{R}^{n}\right)$, then for some $v \in L^{p}\left(\mathbb{R}^{n}\right)$ we have $u=\Lambda^{-n / p} v$, where

$$
\widehat{\left(\Lambda^{s} v\right)}(\xi):=\langle\xi\rangle^{s} \hat{v}(\xi), \quad s \in \mathbb{R}, \quad\langle\xi\rangle:=\left(1+|\xi|^{2}\right)^{1 / 2},
$$

and hat stands for the Fourier transform. Hence,

$$
u=\mathcal{G}_{n / p} * v,
$$

where $\mathcal{G}_{s}$ is the usual Bessel kernel, i.e.

$$
\widehat{\mathcal{G}}_{s}(\xi):=\langle\xi\rangle^{-s}, \quad \xi \in \mathbb{R}^{n} .
$$

The behavior of $\mathcal{G}_{s}$ follows from results in [34, p. 132]. In particular $\mathcal{G}_{s}$ is $C^{\infty}$ on $\mathbb{R}^{n} \backslash\{0\}$ and vanishes rapidly as $|x| \rightarrow \infty$. For $|x| \leq 1$ and $0<s<n$, we also have

$$
\left|\mathcal{G}_{s}(x)\right| \leq C|x|^{s-n} .
$$

This is a key ingredient. Consequently, $\mathcal{G}_{n / p}$ just misses being in $L^{p /(p-1)}\left(\mathbb{R}^{n}\right)$. With this at hand, the estimate (2.19) follows much as in $[\mathbf{3 5}]$; we omit the details.

As for (2.20), we shall use a standard interpolation inequality (cf. [32, Remark 2, p. 87]), to the effect that for each fixed $1<p<\tau<\infty$, and $u \in C_{\text {comp }}^{\infty}(\Omega)$,

$$
\begin{aligned}
\|u\|_{L_{2 / q_{\tau}}^{q_{\tau}}(\Omega)} & \leq\|u\|_{L_{n / p}^{p}(\Omega)}^{1-\theta_{\tau}}\|u\|_{L^{\tau}(\Omega)}^{\theta_{\tau}}, \\
& \text { where } \theta_{\tau}:=\frac{n-2}{n-2+2 p / \tau} \text { and } \frac{1}{q_{\tau}}:=\frac{\theta_{\tau}}{\tau}+\frac{1-\theta_{\tau}}{p} .
\end{aligned}
$$

Notice that, as $\tau \rightarrow \infty$, we have $\theta_{\tau} \rightarrow 1, q_{\tau} \rightarrow \infty$ and $\tau / q_{\tau} \rightarrow n /(n-2)$. Also, $\theta_{\tau} \rightarrow 0$ and $q_{\tau} \rightarrow p$ as $\tau \rightarrow 0$.

Using (2.19) in order to estimate the last factor in (2.25) produces

$$
\|u\|_{L_{2 / q_{\tau}}^{q_{\tau}}(\Omega)} \leq C \tau^{\theta_{\tau}(1-1 / p)}\|u\|_{L_{n / p}^{p}(\Omega)} \leq C q_{\tau}^{1-1 / p}\|u\|_{L_{n / p}^{p}(\Omega)},
$$

where the last inequality is a simple consequence of $\tau^{\theta_{\tau}} \leq C q_{\tau}$. Formally replacing $q_{\tau}$ by $\tau$ in $(2.26)$ (recall that $q_{\tau}$ covers the interval $(p, \infty)$ as $\tau$ ranges from $p$ to $\infty)$, we arrive at

$$
\|u\|_{L_{2 / \tau}^{\tau}(\Omega)} \leq C \tau^{1-1 / p}\|u\|_{L_{n / p}^{p}(\Omega)}, \quad \tau>p, \quad \forall u \in C_{\mathrm{comp}}^{\infty}(\Omega) .
$$


In turn, this readily implies $(2.20)$ given that $L_{2 / \tau, 0}^{\tau}(\Omega)$ and $L_{n / p, 0}^{p}(\Omega)$ are, respectively, the closure of $C_{\mathrm{comp}}^{\infty}(\Omega)$ in $L_{2 / \tau}^{\tau}(\Omega)$ and $L_{n / p}^{p}(\Omega)$ (here we need the condition that $n-1<p<\infty)$. This concludes the proof of the lemma.

Next we initiate the

Proof of Theorem 2.1: Part I: In this first segment, we deal with the well posedness aspect of (2.4), including the accompanying estimate (2.5), in the case when $r>n / 2$.

To this end, assume first that $n \geq 3$ and consider the mappings

$$
\begin{aligned}
& \text { Dir, } R: L_{s+\frac{1}{p}}^{p}(\Omega) \longrightarrow L_{s+\frac{1}{p}-2}^{p}(\Omega) \times B_{s}^{p, p}(\partial \Omega), \\
& \text { Dir } u:=(\Delta u, \operatorname{Tr} u), \quad R u:=(-V u, 0) .
\end{aligned}
$$

Granted the current assumptions, from [30] we know that the Dirichlet Laplacian Dir is an isomorphism while, from Lemma $2.2, R$ is compact. It follows that Dir $+R$ is Fredholm with index zero and we claim that this operator is, in fact, an isomorphism.

Clearly, to see this, it suffices to prove that $\mathrm{Dir}+R$ is one-to-one, a task which we now consider. To see this, assume that $u \in L_{s+\frac{1}{p}}^{p}(\Omega)$ is such that $(\operatorname{Dir}+R) u=0$. Since $u=p r_{1}\left[\operatorname{Dir}^{-1}(V u)\right]$ (here and elsewhere, $p r_{1}$ denotes the natural projection onto the first factor of a Cartesian product), repeated applications of (2.14) eventually show that

$$
u \in \bigcap_{(s, 1 / p) \in \mathcal{H}} L_{s+1 / p, 0}^{p}(\Omega),
$$

where $\mathcal{H}$ is the (hexagonal) region described by (2.2); cf. Figure 1. Next, (2.29) can be used to justify the integration by parts formula

$$
\iint_{\Omega}|\nabla u|^{2}+V|u|^{2}=0
$$

which, in turn, readily entails $u=0$ in $\Omega$. This takes care of the claim made in the previous paragraph and, hence, finishes the proof of the well posedness of (2.4).

At this stage we turn our attention to (2.6). The proof of this estimate is involved and requires a number of auxiliary tools which we now begin to develop in a sequence of lemmas. Final arguments are presented in the last part of this section. 
To get started, if $X, Y$ are two Banach spaces we denote by $\mathcal{L}(X, Y)$ the collection of all linear, bounded operators mapping $X$ into $Y$, and equip it with the usual (strong) operator norm. This space will be further abbreviated as $\mathcal{L}(X)$ when $X=Y$.

We debut with an interpolation result. In view of the fact that $\left\{L_{s, 0}^{p}(\Omega)\right\}_{\substack{1<p<\infty \\ s \geq 0}}$ is a complex interpolation scale, it is natural to ask if for $1<p<\infty$ and $1 / p<s<1+1 / p, 0<\theta<1$ one has

$$
\begin{aligned}
& {\left[L_{s, 0}^{p}(\Omega), L^{\infty}(\Omega)\right]_{\theta}=L_{s^{*}, 0}^{p^{*}}(\Omega),} \\
& \quad \text { where } \frac{1}{p^{*}}=\frac{1-\theta}{p}, s^{*}=(1-\theta) s .
\end{aligned}
$$

Since it is unclear whether the current literature on interpolation covers (2.31), in the lemma below we devise a convenient substitute, equally effective for the applications we have in mind.

Lemma 2.5. Assume that for some $1 \leq q_{0}<\infty$, we have that $T \in$ $\mathcal{L}\left(L^{q}(\Omega)\right)$ for each $q_{0} \leq q \leq \infty$ and, in addition, that $T \in \mathcal{L}\left(L_{s, 0}^{p}(\Omega)\right)$ for some $1<p<\infty, 1 / p<s<1+1 / p$. Then, for each $\theta \in(0,1)$, $T \in \mathcal{L}\left(L_{s^{*}, 0}^{p^{*}}(\Omega)\right)$ where $1 / p^{*}=(1-\theta) / p, s^{*}=(1-\theta) s$, and

$$
\|T\|_{\mathcal{L}\left(L_{s^{*}, 0}^{p^{*}}(\Omega)\right)} \leq\|T\|_{\mathcal{L}\left(L^{\infty}(\Omega)\right)}^{\theta}\|T\|_{\mathcal{L}\left(L_{s, 0}^{p}(\Omega)\right)}^{1-\theta} .
$$

Proof: For each $t<\infty$ large, $T$ belongs to $\mathcal{L}\left(L^{t}(\Omega)\right)$ and there exist two finite constants $K, \kappa>0$ so that

$$
\|T\|_{\mathcal{L}\left(L^{t}(\Omega)\right)} \leq K^{1 / t}\|T\|_{\mathcal{L}\left(L^{\infty}(\Omega)\right)}^{1-\kappa / t} .
$$

Set $p_{t}:=((1-\theta) / p+\theta / t)^{-1}$. Since $\left[L_{s, 0}^{p}(\Omega), L^{t}(\Omega)\right]_{\theta}=L_{s^{*}, 0}^{p_{t}}(\Omega)$, it follows that

$$
\|T\|_{\mathcal{L}\left(L_{s^{*}, 0}^{p_{t}}(\Omega)\right)} \leq\|T\|_{\mathcal{L}\left(L_{s, 0}^{p}(\Omega)\right)}^{1-\theta} K^{\theta / t}\|T\|_{\mathcal{L}\left(L^{\infty}(\Omega)\right)}^{\theta(1-\kappa / t)} .
$$

Thus, for each $f \in C_{\text {comp }}^{\infty}(\Omega)$, we may write

$$
\begin{aligned}
\|T f\|_{L_{s^{*}, 0}^{p^{*}}(\Omega)} & \leq \lim _{t \rightarrow \infty} \sup \|T f\|_{L_{s^{*}, 0}^{p_{t}}(\Omega)} \\
& \leq \lim _{t \rightarrow \infty} \sup \|f\|_{L_{s^{*}, 0}^{p_{t}}(\Omega)}\|T\|_{\mathcal{L}\left(L_{s^{*}, 0}^{p_{t}}(\Omega)\right)} \\
& \leq\|f\|_{L_{s^{*}, 0}^{p^{*}}(\Omega)}\|T\|_{\mathcal{L}\left(L_{s, 0}^{p}(\Omega)\right)_{t \rightarrow \infty}}^{1-\theta} \lim \sup K^{\theta / t}\|T\|_{\mathcal{L}\left(L^{\infty}(\Omega)\right)}^{\theta(1-\kappa / t)} \\
& =\|f\|_{L_{s^{*}, 0}^{p^{*}}(\Omega)}\|T\|_{\mathcal{L}\left(L_{s, 0}^{p}(\Omega)\right)}^{1-\theta}\|T\|_{\mathcal{L}\left(L^{\infty}(\Omega)\right)}^{\theta}
\end{aligned}
$$

This, in turn, readily yields the desired conclusion. 
Hereafter, we shall denote by $(\Delta-V)_{D}^{-1}$ the solution operator for the Poisson problem with homogeneous Dirichlet boundary conditions. In other words, $u:=(\Delta-V)_{D}^{-1} f$ is the solution of the Poisson problem $(\Delta-V) u=f$ in $\Omega, \operatorname{Tr} u=0$ on $\partial \Omega$.

In order to continue, we recall some useful facts about fractional powers of the Dirichlet Laplacian in Lipschitz domains. These have been proved in $[\mathbf{2 4}]$, [30] (cf. also [26] for $n=2$ ), following earlier work in $[\mathbf{1 6}]$.

Proposition 2.6. Let $\Omega \subset M$ be an arbitrary Lipschitz domain and denote by $A:=(-\Delta)_{D}^{-1 / 2}$, the inverse of the square-root of the (negative of the) Dirichlet Laplacian in $\Omega$. Then

$$
A^{\mu}: L^{p}(\Omega) \stackrel{\sim}{\longrightarrow} L_{\mu, 0}^{p}(\Omega)
$$

is an isomorphism provided

$$
\max \left\{\frac{\mu}{3}-\varepsilon, \mu-1\right\} \leq \frac{1}{p}<1, \quad 0 \leq \mu \leq 2, \text { if } n \geq 3,
$$

or

$$
\max \left\{\frac{\mu}{2}-\frac{1}{2}-\varepsilon, \mu-1\right\} \leq \frac{1}{p}<1, \quad 0 \leq \mu \leq 2, \text { if } n=2,
$$

for some $\varepsilon=\varepsilon(\partial \Omega)>0$. When $\partial \Omega \in C^{1}$, one can simply require that $\mu \geq 0$ together with $\max \{0, \mu-1\} \leq 1 / p<1$.

Next, if $\Omega$ is a Lipschitz domain and $V \geq 0, V \in L^{r}(\Omega)$, we consider the operator

$$
T_{V}:=(\Delta-V)_{D}^{-1} \Delta .
$$

From what we have proved so far, for each $V \in L^{r}(M), r>n / 2, V \geq 0$, $T_{V}$ is a well defined, bounded mapping of $L_{s+1 / p}^{p}(\Omega)$ into itself for each $(s, 1 / p)$ as in (2.2) if $n \geq 3$, or as in (2.10) if $n=2$, as long as (2.3) is satisfied. Our immediate goal is to establish estimates on the norm of the operator $T_{V}$ uniformly in $V$. As we shall see momentarily, Lemma 2.5 and Proposition 2.6 are two key ingredients in this regard.

Lemma 2.7. Let $\Omega$ be an $n$-dimensional Lipschitz domain, $n \geq 2$, $1<p<\infty$, and assume that the function $V$ is as above. Then $T_{V}$ : $L_{2 / p, 0}^{p}(\Omega) \rightarrow L_{2 / p, 0}^{p}(\Omega)$ satisfies

$$
\left\|T_{V}\right\|_{\mathcal{L}\left(L_{2 / p, 0}^{p}(\Omega)\right)} \leq C
$$

where $C=C(\Omega, p)>0$ is a finite constant independent of $V$. 
Proof: In broad outline, the plan is to prove first that the estimates

$$
\left\|T_{V}\right\|_{\mathcal{L}\left(L_{1,0}^{2}(\Omega)\right)} \leq C, \quad\left\|T_{V}\right\|_{\mathcal{L}\left(L^{\infty}(\Omega)\right)} \leq C
$$

hold uniformly with respect to the function $V$, and then to use (2.41) in conjunction with Lemma 2.5 in order to obtain (2.40) when $2 \leq p<\infty$. The situation when $1<p<2$ is then handled via a duality argument which makes use of Proposition 2.6.

Turning to specifics, the first estimate in (2.41) is seen as follows. If $f \in L_{1,0}^{2}(\Omega)$ and we set $u:=T_{V} f=(\Delta-V)_{D}^{-1}(\Delta f)$, then $u$ is a solution of

$$
(\Delta-V) u=\Delta f \text { in } \Omega, \quad \operatorname{Tr} u=0 \text { on } \partial \Omega .
$$

Now, a simple argument which involves pairing both sides of (2.42) with $u$ and integrating by parts gives

$$
\begin{aligned}
\|u\|_{L_{1,0}^{2}(\Omega)}^{2} & \leq C \iint_{\Omega}|\nabla u|^{2} \leq C \iint_{\Omega}|\nabla u|^{2}+V|u|^{2}=C\langle-\Delta f, u\rangle \\
& \leq C\|\Delta f\|_{L_{-1,0}^{2}(\Omega)}\|u\|_{L_{1,0}^{2}(\Omega)} \leq C\|f\|_{L_{1,0}^{2}(\Omega)}\|u\|_{L_{1,0}^{2}(\Omega)},
\end{aligned}
$$

where all the constants above depend only on the domain $\Omega$. Clearly, (2.43) yields the desired estimate.

The proof of the second estimate in (2.41) is more challenging. Denote by $\dot{T}_{V}$ the restriction of the operator $(2.39)$ to $L_{s+1 / p, 0}^{p}(\Omega)$, which maps this space isomorphically onto itself (granted the well-posedness of the Poisson problem (2.4)). Thus,

$$
\dot{T}_{V} f=\left(I+(\Delta-V)_{D}^{-1} V\right) f=f+(\Delta-V)_{D}^{-1}(V f) .
$$

We also find it useful working with the operator $\dot{T}_{V}^{-1}$ which, in the same context, can be written as

$$
\dot{T}_{V}^{-1} f=\left(I-\Delta_{D}^{-1} V\right) f=f-\Delta_{D}^{-1}(V f) .
$$

Consider the class $C_{0}(\Omega):=\left\{f \in C(\bar{\Omega}) ;\left.f\right|_{\partial \Omega}=0\right\}$, equipped with the usual supremum norm. A standard mollifier argument shows that $C_{\text {comp }}^{\infty}(\Omega) \hookrightarrow C_{0}(\Omega)$ densely. Our first claim is that $\dot{T}_{V}, \dot{T}_{V}^{-1}$, defined in (2.44)-(2.45), extend as isomorphisms of $C_{0}(\Omega)$.

To this end, observe that $f \in C_{0}(\Omega)$ entails $V f \in L^{r}(\Omega)$ and, further, $V f \in L_{s+1 / p-2}^{p}(\Omega)$ if

$$
\frac{2}{n}-\frac{1}{r} \geq-\frac{1}{p}+\frac{s+1 / p}{n} .
$$


Assuming that this is the case and granted that $s, p$ are such that the Poisson problem (2.4) is well-posed, we get $(\Delta-V)_{D}^{-1}(V f), \Delta_{D}^{-1}(V f) \in$ $L_{s+1 / p}^{p}(\Omega) \hookrightarrow C^{\varepsilon}(\bar{\Omega})$, provided

$$
-\frac{1}{p}+\frac{s+1 / p}{n}>0 \text {, and } \varepsilon>0 \text { is small. }
$$

Since both (2.46) and (2.47) can be satisfied for some pair $s, p$ within the well-posedness range for the Poisson problem (2.4), we may conclude that both $\dot{T}_{V}$ and $\dot{T}_{V}^{-1}$ are well defined mappings of $C_{0}(\Omega)$ into itself. Since $\dot{T}_{V} \circ \dot{T}_{V}^{-1}=\dot{T}_{V}^{-1} \circ \dot{T}_{V}=I$ on $C_{\text {comp }}^{\infty}(\Omega)$, the desired conclusion follows.

In a similar fashion, one can check that

$$
\begin{aligned}
\left\|\dot{T}_{V} f\right\|_{L^{p}(\Omega)},\left\|\dot{T}_{V}^{-1} f\right\|_{L^{p}(\Omega)} \leq & C(V)\|f\|_{L^{p}(\Omega)}, \\
& \forall f \in C_{0}(\Omega), \text { if } \frac{r}{r-1} \leq p \leq \infty .
\end{aligned}
$$

Thus, $\dot{T}_{V}, \dot{T}_{V}^{-1}$ from (2.44), (2.45) can also be extend as isomorphisms of $L^{p}(\Omega)$, for $\frac{r}{r-1} \leq p<\infty$.

Next, we aim at showing that for any $V \in L^{r}(\Omega), V \geq 0$, with $r>n / 2$ $(n \geq 2)$ there holds

$$
\left\|\dot{T}_{V} f\right\|_{L^{\infty}(\Omega)} \leq 2\|f\|_{L^{\infty}(\Omega)}, \quad \text { uniformly for } f \in L^{\infty}(\Omega) .
$$

With an eye on (2.49), we shall now argue to the effect that for each $V$ as above,

$$
\frac{1}{2}\|f\|_{L^{\infty}(\Omega)} \leq\left\|\dot{T}_{V}^{-1} f\right\|_{L^{\infty}(\Omega)}, \quad \text { uniformly for } f \in C_{0}(\Omega) .
$$

Seeking a contradiction, assume the contrary, i.e., that (2.50) is false. Then we can find a nonnegative function $V \in L^{r}(\Omega)$ and $f \in C_{0}(\Omega)$ such that

$$
\frac{1}{2}\|f\|_{L^{\infty}(\Omega)}>\left\|\dot{T}_{V}^{-1} f\right\|_{L^{\infty}(\Omega)} .
$$

Without loss of generality we can assume that $\sup _{x \in \Omega} f(x)=\|f\|_{L^{\infty}(\Omega)}$, otherwise work with the function $-f$ instead. Since $f$ is continuous and zero at the boundary, it attains its maximum at some point $x^{*} \in \Omega$, i.e. $f\left(x^{*}\right)=\|f\|_{L^{\infty}(\Omega)}$. Denote by $\mathcal{O}$ the connected component of the open set $\{x \in \Omega ; f(x)>0\}$ which contains the point $x^{*}$, and let $u:=$ $\Delta_{D}^{-1}(V f) \in C_{0}(\Omega)$. By (2.51) we get

$$
\frac{1}{2} f\left(x^{*}\right)>\left|f\left(x^{*}\right)-u\left(x^{*}\right)\right|,
$$


hence $u\left(x^{*}\right)>\frac{1}{2} f\left(x^{*}\right)>0$. Observe that the function $u$ is a supersolution for the Laplace-Beltrami operator on $\Omega$ (since $\Delta u=V f \geq 0$ in $\Omega$ ) so that, when considered on $\mathcal{O}$, it must attain its maximum at a boundary point. Thus, there exists $z \in \partial \mathcal{O} \subset \Omega$ at which $u(z)=\max _{x \in \mathcal{O}} u(z) \geq$ $u\left(x^{*}\right)>\frac{1}{2} f\left(x^{*}\right)$. On the other hand, the definition of $\mathcal{O}$ ensures that $f \equiv 0$ on $\partial \mathcal{O}$. Consequently, $\dot{T}_{V}^{-1} f(z)=f(z)-u(z)<-\frac{1}{2} f\left(x^{*}\right)<0$, so that $\left|\dot{T}_{V}^{-1} f(z)\right|>\frac{1}{2}\|f\|_{L^{\infty}(\Omega)}$. This contradicts (2.51), and concludes the proof of (2.50).

Turning now to the task of proving (2.49), recall that for each $r /(r-1) \leq p \leq \infty$, there exists a constant $\kappa(V)>0$, independent of $p$, such that

$$
\left\|\dot{T}_{V} f\right\|_{L^{p}(\Omega)} \leq \kappa(V)\|f\|_{L^{p}(\Omega)} \text { and }\left\|\dot{T}_{V} f\right\|_{L^{\infty}(\Omega)} \leq 2\|f\|_{L^{\infty}(\Omega)},
$$

uniformly for $f \in C_{0}(\Omega)$. A standard interpolation inequality (cf., e.g., $\left[13\right.$, p. 41]) then yields, for $f \in C_{0}(\Omega)$ and $p \in[r /(r-1), \infty]$,

$$
\begin{aligned}
\left\|\dot{T}_{V} f\right\|_{L^{p}(\Omega)} & \leq\left\|\dot{T}_{V} f\right\|_{L^{r /(r-1)}(\Omega)}^{\theta}\left\|\dot{T}_{V} f\right\|_{L^{\infty}(\Omega)}^{1-\theta} \\
& \leq \kappa(V)^{\theta} 2^{1-\theta}\|f\|_{L^{r /(r-1)}(\Omega)}^{\theta}\|f\|_{L^{\infty}(\Omega)}^{1-\theta},
\end{aligned}
$$

where $\theta:=r /(p(r-1)) \in(0,1)$. In particular, $\theta \searrow 0$ as $p \nearrow \infty$.

Now, given any $f \in L^{\infty}(\Omega)$, we can find a sequence of functions $\left(f_{j}\right)_{j}$ in $C_{0}(\Omega)$ such that $\left\|f_{j}\right\|_{L^{\infty}(\Omega)} \leq\|f\|_{L^{\infty}(\Omega)}$ and $f_{j} \rightarrow f$ in $L^{q}(\Omega)$ for any $q<\infty$. Then, by virtue of $(2.54)$, we get

$$
\begin{aligned}
\left\|T_{V} f\right\|_{L^{\infty}(\Omega)} & =\lim _{p \rightarrow \infty}\left\|T_{V} f\right\|_{L^{p}(\Omega)}=\lim _{p \rightarrow \infty} \lim _{j \rightarrow \infty}\left\|\dot{T}_{V} f_{j}\right\|_{L^{p}(\Omega)} \\
& \leq \lim _{p \rightarrow \infty} \lim _{j \rightarrow \infty} \kappa(V)^{\theta} 2^{1-\theta}\left\|f_{j}\right\|_{L^{r /(r-1)}(\Omega)}^{\theta}\left\|f_{j}\right\|_{L^{\infty}(\Omega)}^{1-\theta} \\
& \leq 2\|f\|_{L^{\infty}(\Omega)} .
\end{aligned}
$$

This justifies (2.49) and shows that $\left\|T_{V}\right\|_{\mathcal{L}\left(L^{\infty}(\Omega)\right)} \leq 2$, independently of $V$, thus proving (2.41).

With (2.41) in hand and relying on Lemma 2.5, we see that (2.40) holds for each $2 \leq p<\infty$, uniformly in $V$.

There remains the case $1<p<2$ which we treat next. Our strategy is based on duality and requires analyzing the action of the operator

$$
K_{V, p}:=(-\Delta)_{D}^{1 / p} T_{V}(-\Delta)_{D}^{-1 / p}=-(-\Delta)_{D}^{1 / p}(\Delta-V)_{D}^{-1}(-\Delta)_{D}^{1-1 / p}
$$

on $L^{p}$ spaces. Concretely, from Proposition 2.6 (with $\mu=2 / p$ ) it suffices to show that $\left\|K_{V, p}\right\|_{\mathcal{L}\left(L^{p}(\Omega)\right)}$ is bounded uniformly in $V$ or, equivalently, 
that $\left\|K_{V, p}^{*}\right\|_{\mathcal{L}\left(L^{p^{\prime}}(\Omega)\right)} \leq C(p)$ uniformly in $V$, where $1 / p+1 / p^{\prime}=1$. Since

$$
\begin{aligned}
K_{V, p}^{*} & =-(-\Delta)_{D}^{1-1 / p}(\Delta-V)_{D}^{-1}(-\Delta)_{D}^{1 / p} \\
& =(-\Delta)_{D}^{1 / p^{\prime}} T_{V}(-\Delta)_{D}^{-1 / p^{\prime}}=K_{V, p^{\prime}}
\end{aligned}
$$

by relying once again on Proposition 2.6, we see that matters are further reduced to proving that $\left\|T_{V}\right\|_{\mathcal{L}\left(L_{2 / p^{\prime}, 0}^{p^{\prime}}(\Omega)\right)} \leq C$ uniformly in $V$. However, having $p \in(1,2)$ entails $p^{\prime} \in(2, \infty)$ and this is precisely the case already addressed. The proof of the lemma is therefore finished.

In our next lemma we consider the action of the operator $T_{V}$ on $L^{p}$ spaces.

Lemma 2.8. Assume that $n \geq 3$. Then, if $V \in L^{r}(\Omega), r>n / 2$, is nonnegative, there holds

$$
\left\|T_{V}\right\|_{\mathcal{L}\left(L^{p}(\Omega)\right)} \leq C\left(1+\|V\|_{L^{r}(\Omega)}\right) \quad \text { for each } p>r /(r-1),
$$

where $C=C(\Omega, r, p)>0$ is independent of $V$. The estimate (2.58) also holds when $n=2$ provided $r>4 / 3$.

Proof: Assume first that $n \geq 3$. To prove (2.58), we rely on the fact that $T_{V} f=f+(\Delta-V)_{D}^{-1}(V f)$ for $f \in C_{\mathrm{comp}}^{\infty}(\Omega)$, and analyze the actions of the multiplicative operator $M_{V}$, defined by $M_{V} f:=V \cdot f$, and $(\Delta-V)_{D}^{-1}$ separately. In this scenario, the crux of the matter is establishing the estimate

$$
\left\|(\Delta-V)_{D}^{-1}\right\|_{\mathcal{L}\left(L^{q}(\Omega), L^{q n /(n-2 q)}(\Omega)\right)} \leq C, \quad q \in(1, n / 2),
$$

for some $C=C(q)>0$ independent of $V$. Indeed, (2.59) in concert with the elementary estimate $\left\|M_{V}\right\|_{\mathcal{L}\left(L^{p}(\Omega), L^{p r /(p+r)}(\Omega)\right)} \leq\|V\|_{L^{r}(\Omega)}$, readily yields (2.58) provided that one can choose $q \in(1, n / 2)$ so that $1 / r+1 / p \leq$ $1 / q \leq 1 / p+2 / n$. As this latter condition is easily checked, (2.58) will follow as soon as we justify (2.59).

In this regard, a simple yet useful observation (whose proof amounts to an algebra exercise) is that

(2.60) $\forall q \in(1, n / 2)$,

$$
\exists(s, 1 / p) \text { as in }(2.2) \text { such that } L^{q}(\Omega) \hookrightarrow L_{s+1 / p-2}^{p}(\Omega) .
$$

Consequently, one can always employ a factorization of the type

$$
\begin{aligned}
(\Delta-V)_{D}^{-1}: L^{q}(\Omega) \stackrel{\iota}{\longrightarrow} L_{s+1 / p-2}^{p}(\Omega) \stackrel{(\Delta-V)_{D}^{-1}}{\longrightarrow} L_{s+1 / p, 0}^{p}(\Omega) \\
\stackrel{\iota}{\longrightarrow} L^{q n /(n-2 q)}(\Omega)
\end{aligned}
$$


in order to justify that $(\Delta-V)_{D}^{-1} \in \mathcal{L}\left(L^{q}(\Omega), L^{q n /(n-2 q)}(\Omega)\right)$ for each $q \in(1, n / 2)$.

Turning now to the actual task of proving (2.59), fix an arbitrary $f \in L^{q}(\Omega)$ which we write as $f=f^{+}-f^{-}$, where $0 \leq f^{+}, f^{-} \leq|f|$. Positivity of $(-\Delta)_{D}^{-1}$ gives

$$
0 \leq f^{ \pm} \leq V(-\Delta)_{D}^{-1} f^{ \pm}+f^{ \pm}
$$

Applying $(V-\Delta)_{D}^{-1}$ to the above inequalities and invoking Lemma 2.3 yields

$$
0 \leq(V-\Delta)_{D}^{-1} f^{ \pm} \leq(-\Delta)_{D}^{-1} f^{ \pm} .
$$

Together with the decomposition $f=f^{+}-f^{-}$and (2.61) with $V=0$, this allows us to write

$$
\begin{aligned}
\left\|(V-\Delta)_{D}^{-1} f\right\|_{L^{q n /(n-2 q)}(\Omega)} \leq & \left\|(-\Delta)_{D}^{-1} f^{+}\right\|_{L^{q n /(n-2 q)}(\Omega)} \\
& +\left\|(-\Delta)_{D}^{-1} f^{-}\right\|_{L^{q n /(n-2 q)}(\Omega)} \\
\leq & 2 C\|f\|_{L^{q}(\Omega)},
\end{aligned}
$$

as desired. This establishes (2.59) and finishes the proof of (2.58) when $n \geq 3$.

When $n=2$ one can proceed in a similar fashion, the most notable difference being that (2.59) now becomes $\left\|(\Delta-V)_{D}^{-1}\right\|_{\mathcal{L}\left(L^{q}(\Omega), L^{\infty}(\Omega)\right)} \leq$ $C$, for $q>1$.

Armed with Lemma 2.7 and Lemma 2.8 we now turn to the task of establishing the following important estimates.

Lemma 2.9. Assume that $V \in L^{r}(\Omega), r>n / 2$, is an arbitrary nonnegative function, and that $0<s<1,1<p<\infty$.

(i) If $s /(n-1)<1 / p \leq s$, then

$$
\left\|T_{V}\right\|_{\mathcal{L}\left(L_{s+1 / p, 0}^{p}(\Omega), L^{n p /(n-1-s p)}(\Omega)\right)} \leq C,
$$

where $C=C(\Omega, s, p)>0$ is a finite constant independent of $V$.

(ii) If $s p>n-1$ then, for some constant $C=C(\Omega, r, p, s)>0$ independent of the function $V$,

$$
\left\|T_{V}\right\|_{\mathcal{L}\left(L_{s+1 / p, 0}^{p}(\Omega), L^{\infty}(\Omega)\right)} \leq C .
$$

(iii) If $s p=n-1$, there exists $C=C(\Omega, r, p)>0$ independent of $V$ such that

$$
\left\|T_{V}\right\|_{\mathcal{L}\left(L_{s+1 / p, 0}^{p}(\Omega), L^{\tau}(\Omega)\right)} \leq C \tau^{1-1 / p} \quad \text { for any } \tau \in[2, \infty) .
$$


(iv) If $s<\frac{1}{p}<\frac{s}{n-1}+\frac{n(r-1)}{r(n-1)}$, $n \geq 3$, then

(2.68) $\left\|T_{V}\right\|_{\mathcal{L}\left(L_{s+1 / p, 0}^{p}(\Omega), L^{n p /(n-1-s p)}(\Omega)\right)}$

$\leq \begin{cases}C\left(1+\|V\|_{L^{r}(\Omega)}\right)^{\varepsilon+\frac{r}{2(r-1)}\left(\frac{1}{p}-s\right)}, & \text { if } r \leq 1+(1-s p) /(2 p-2), \\ C\left(1+\|V\|_{L^{r}(\Omega)}\right)^{\varepsilon+\frac{1}{2-n / r}((n-1) / p-s-n+2)}, & \text { if } r<1+(1-s p) /(2 p-2),\end{cases}$

for each $\varepsilon>0$, where $C=C(\Omega, s, p, \varepsilon)>0$ is independent of $V$. When $n=2$, the same holds provided $r>4 / 3$.

Proof: Consider first the situation when $0<s /(n-1)<1 / p \leq s<1$ (which automatically entails $n \geq 3$ ). The case $s p=1,1<p<\infty$ has been dealt with in Lemma 2.7. The larger range we intend to addressed here is then a simple consequence of this and the factorization

(2.69) $T_{V}: L_{s+1 / p, 0}^{p}(\Omega) \hookrightarrow L_{2 / p^{*}, 0}^{p^{*}}(\Omega) \stackrel{T_{V}}{\longrightarrow} L_{2 / p^{*}, 0}^{p^{*}}(\Omega) \hookrightarrow L^{n p /(n-1-s p)}(\Omega)$,

where $1 / p^{*}:=(n-1)(1 / p-s /(n-1)) /(n-2)$, and both inclusions above are classical embedding results. As for (ii), i.e. the case $s p>n-1, n \geq 2$, we use (2.41) and the factorization

$$
T_{V}: L_{s+1 / p, 0}^{p}(\Omega) \hookrightarrow L^{\infty}(\Omega) \stackrel{T_{V}}{\longrightarrow} L^{\infty}(\Omega) .
$$

Next we address the case $s p=n-1$ (which forces $p>n-1$ ). When $n=2$, the conclusion we seek is an immediate consequence of Lemma 2.7 and Lemma 2.4. Similar ingredients can be used to handle the case $n \geq 3$ as well. The idea is to use the factorization

$$
T_{V}: L_{n / p, 0}^{p}(\Omega) \stackrel{\iota_{1}}{\longrightarrow} L_{2 / \tau, 0}^{\tau}(\Omega) \stackrel{T_{V}}{\longrightarrow} L_{2 / \tau, 0}^{\tau}(\Omega) \stackrel{\iota_{2}}{\longleftrightarrow} L^{\tau}(\Omega),
$$

in concert with $\left\|\iota_{1}\right\| \leq C \tau^{1-1 / p},(2.40)$, and the fact that $\left\|\iota_{2}\right\| \leq C$, uniformly in $\tau \in[p, \infty)$. This finishes the analysis of the point (iii) in our lemma.

At this stage, it remains to deal with the situation described in the point (iv) of the lemma, and our intention is to eventually interpolate between (2.40) and (2.58). To this end, recall first that the two classes, $L_{s+1 / p, 0}^{p}(\Omega)$ and $L^{n p /(n-1-s p)}(\Omega)$, with $1<p<\infty$ and $-1 / p \leq s$, are complex interpolation scales, in the sense that for each $1<p_{j}<\infty$, 
$s_{j} \geq-1 / p_{j}, j=0,1$,

$$
\begin{aligned}
& {\left[L_{s_{0}+1 / p_{0}, 0}^{p_{0}}(\Omega), L_{s_{1}+1 / p_{1}, 0}^{p_{1}}(\Omega)\right]_{\theta}=L_{s^{*}+1 / p^{*}, 0}^{p^{*}}(\Omega),} \\
& {\left[L^{n p_{0} /\left(n-1-s_{0} p_{0}\right)}(\Omega), L^{n p_{1} /\left(n-1-s_{1} p_{1}\right)}(\Omega)\right]_{\theta}=L^{n p^{*} /\left(n-1-s^{*} p^{*}\right)}(\Omega),}
\end{aligned}
$$

$$
\text { if } 1 / p^{*}:=(1-\theta) / p_{0}+\theta / p_{1}, s^{*}:=(1-\theta) s_{0}+\theta s_{1}, 0<\theta<1 \text {. }
$$

This observation and convexity arguments readily entail the following principle: if $1<p_{i}<\infty,-1 / p_{i} \leq s_{i}, \tau_{i}:=n p_{i} /\left(n-1-s_{i} p_{i}\right)>1$, and $\left\|T_{V}\right\|_{\mathcal{L}\left(L_{s_{i}+1 / p_{i}}^{p_{i}}(\Omega), L^{\tau_{i}}(\Omega)\right)} \leq M_{i}, i=1,2,3$, then

$$
\left\|T_{V}\right\|_{\mathcal{L}\left(L_{s+1 / p, 0}^{p}(\Omega), L^{n p /(n-1-s p)}(\Omega)\right)} \leq M_{1}^{\lambda_{1}} M_{2}^{\lambda_{2}} M_{3}^{\lambda_{3}},
$$

provided the point $(s, 1 / p)$ has baricentrical coordinates $\left(\lambda_{1}, \lambda_{2}, \lambda_{3}\right)$, $\lambda_{i} \geq 0$, relative to the triangle with vertices $\left(s_{i}, 1 / p_{i}\right), i=1,2,3$.

We shall use the above remark twice, first for the triangle with vertices at $(\varepsilon, \varepsilon),(1-\varepsilon, 1-\varepsilon),(1 /(r+\varepsilon)-1,1-1 /(r+\varepsilon))$ with $\varepsilon>0$ sufficiently small. In this scenario, assuming that $n \geq 3, M_{1}$ and $M_{2}$ are controlled by $C_{\varepsilon}$ and $M_{3} \leq C_{\varepsilon}\left(1+\|V\|_{L^{r}(\Omega)}\right)$. Also, $\lambda_{3}$ is a $O(\varepsilon)$ variation of $r(1 / p-s) /(2(r-1))$. This yields the first line in (2.68). The condition $r>1+(1-s p) /(2 p-2)$ guarantees that the point $(s, 1 / p)$ lies inside the triangle under discussion.

The second application of the aforementioned remark is similar in spirit and requires a preparatory step. Specifically, as a result of (2.58) and the factorization

$$
T_{V}: L_{s+1 / p, 0}^{p}(\Omega) \hookrightarrow L^{n p /(n-1-s p)}(\Omega) \stackrel{T_{V}}{\longrightarrow} L^{n p /(n-1-s p)}(\Omega)
$$

it follows that

$$
\begin{aligned}
&\left\|T_{V}\right\|_{\mathcal{L}\left(L_{s+1 / p, 0}^{p}(\Omega), L^{n p /(n-1-s p)}(\Omega)\right)} \leq C\left(1+\|V\|_{L^{r}(\Omega)}\right) \\
& \text { if } 0<\frac{1}{p}-\frac{s}{n-1}<\frac{n(r-1)}{r(n-1)} .
\end{aligned}
$$

Note that the intersection between $\frac{1}{p}-\frac{s}{n-1}=\frac{n(r-1)}{r(n-1)}$ with $1 / p=1$ is the point with coordinates $(n / r-1,1)$. Inspired by this observation, we write (2.73) for the triangle with vertices at $(1-\varepsilon, 1-\varepsilon),(1 /(r+\varepsilon)-1,1-1 /(r+$ $\varepsilon)),(n /(r+\varepsilon)-\varepsilon(n-1)-1,1-\varepsilon)$ for some sufficiently small $\varepsilon>0$. This time, assuming that $n \geq 3$, we get $M_{1} \leq C_{\varepsilon}, M_{2}, M_{3} \leq C_{\varepsilon}\left(1+\|V\|_{L^{r}(\Omega)}\right)$, whereas $\lambda_{2}+\lambda_{3}$ is a $O(\varepsilon)$ variation of $[(n-1) / p-s-n+2] /(2-n / r)$. Availing ourselves of (2.58), (2.40), the second line of (2.68) follows. The 
role of $r \leq 1+(1-s p) /(2 p-2)$ and $\frac{1}{p}-\frac{s}{n-1}<\frac{n(r-1)}{r(n-1)}$ is to ensure that the point $(s, 1 / p)$ lies inside the triangle we are currently considering.

The subcase of (iv) corresponding to $n=2$ is handled similarly, and requires that $r>4 / 3$ (cf. Lemma 2.8).

Finally, we have all the necessary ingredients in order to tackle the

Proof of Theorem 2.1: Part II: Here we present the final arguments in the proof of (2.6), (2.8), (2.7), and also treat the case $r=n / 2$.

In what follows, if $h$ is an arbitrary real-valued function, we set

$$
h^{ \pm}:=\max \{ \pm h, 0\}=\frac{1}{2}(|h| \pm h),
$$

so that $h^{ \pm} \geq 0$ and $h=h^{+}-h^{-}$. Also, $\left|h^{ \pm}(x)-h^{ \pm}(y)\right| \leq|h(x)-h(y)|$ so that

$$
(\cdot)^{ \pm}: B_{s}^{p, p}(\partial \Omega) \longrightarrow B_{s}^{p, p}(\partial \Omega) \text { are bounded, } 1<p<\infty, 0<s<1 .
$$

For the time being, we continue to assume $r>n / 2$. If we now denote by $v_{V}^{( \pm)} \in L_{s+1 / p}^{p}(\Omega)$ the solutions of

$$
L v_{V}^{( \pm)}=(\Delta-V) v_{V}^{( \pm)}=0 \text { in } \Omega, \quad \operatorname{Tr} v_{V}^{( \pm)}=g^{ \pm},
$$

then, clearly, $v_{V}:=v_{V}^{(+)}-v_{V}^{(-)}$solves

$$
L v_{V}=(\Delta-V) v_{V}=0 \text { in } \Omega, \quad \operatorname{Tr} v_{V}=g .
$$

Set also $v_{0}^{( \pm)}$for the solutions of (2.78) with $V=0$. According to Lemma 2.3 we have

$$
0 \leq v_{V}^{(+)} \leq v_{0}^{(+)} \quad \text { and } \quad 0 \leq v_{V}^{(-)} \leq v_{0}^{(-)} .
$$

Assuming $s p<n-1$, it follows that

$$
\begin{aligned}
\left\|v_{V}\right\|_{L^{n p /(n-1-s p)}(\Omega)} & \leq\left\|v_{V}^{(+)}\right\|_{L^{n p /(n-1-s p)}(\Omega)}+\left\|v_{V}^{(-)}\right\|_{L^{n p /(n-1-s p)}(\Omega)} \\
& \leq\left\|v_{0}^{(+)}\right\|_{L^{n p /(n-1-s p)}(\Omega)}+\left\|v_{0}^{(-)}\right\|_{L^{n p /(n-1-s p)}(\Omega)} \\
& \leq C\left\|v_{0}^{(+)}\right\|_{L_{s+1 / p}^{p}(\Omega)}+C\left\|v_{0}^{(-)}\right\|_{L_{s+1 / p}^{p}(\Omega)} \\
& \leq 2 C\|g\|_{B_{s}^{p, p}(\partial \Omega)},
\end{aligned}
$$

where the last constant $C$ in (2.81) is that appearing in the estimate (2.5) for $V=0$. The case when $s p>n-1$ is similar, while the case $s p=n-1$ is proved with the help of Lemma 2.4.

Similarly, by $w_{V}$ we denote the solution to

$$
L w_{V}=(\Delta-V) w_{V}=f \text { in } \Omega, \quad \operatorname{Tr} w_{V}=0 .
$$


Clearly, $w_{0}=\Delta_{D}^{-1} f$ and $w_{V}=(\Delta-V)_{D}^{-1} f$, hence

$$
\begin{aligned}
\left\|w_{V}\right\|_{L^{\tau}(\Omega)} & =\left\|(\Delta-V)_{D}^{-1} \Delta w_{0}\right\|_{L^{\tau}(\Omega)} \\
& \leq\left\|T_{V}\right\|_{\mathcal{L}\left(L_{s+1 / p}^{p}(\Omega), L^{\tau}(\Omega)\right)}\left\|w_{0}\right\|_{L_{s+1 / p}^{p}(\Omega)} \\
& \leq C\left\|T_{V}\right\|_{\mathcal{L}\left(L_{s+1 / p}^{p}(\Omega), L^{\tau}(\Omega)\right)}\|f\|_{L_{s+1 / p-2}^{p}(\Omega)} .
\end{aligned}
$$

The constant $C$ appearing in the last line is again the constant in the estimate (2.5) for $V=0$. Now, $u_{V}=v_{V}+w_{V}$ solves the desired equation (2.4) and the estimates (2.6)-(2.7) follow from (2.81), (2.83) and Lemma 2.9.

In the two dimensional situation, the well posedness range (2.10) is handled analogously, granted the work in [25], [26]. Here we only want to remark that, in order to justify $(2.30)$ for $u \in L_{s+1 / p, 0}^{p}(\Omega)$ and $V \in$ $L^{r}(\Omega), r>(1-|1 /(2 p)-s / 2|)^{-1}$, observe first that for some $\varepsilon>0$ small, $V u \in L^{1+\varepsilon}(\Omega) \hookrightarrow L_{2 / q-2+\varepsilon}^{q}(\Omega)$, if $q>1$. In turn, this further entails $u=$ $p r_{1}\left[\operatorname{Dir}^{-1}(V u)\right] \in L_{2 / q+\varepsilon}^{q}(\Omega) \hookrightarrow L_{1}^{2}(\Omega)$, thanks to the aforementioned references. Since, under the current assumptions, $V|u|^{2} \in L^{1}(\Omega)$ is also readily verified, this takes care of $(2.30)$ when $n=2$.

Finally, we are left with the analyzing the case $r=n / 2$, a task which we take up next. For starters, we claim that it suffices to deal with the situation when the datum $f$ is actually selected from $L^{q}(\Omega)$, where $q$ is given by

$$
\frac{1}{q}:=\frac{2}{n}+\frac{n-1-s p}{n p} .
$$

Indeed, given an arbitrary $f \in L_{s+1 / p-2}^{p}(\Omega)$, set $w:=\Delta_{D}^{-1} f \in L_{s+1 / p, 0}^{p}(\Omega)$. Then $u=u_{0}+w$ solves (2.1), provided $u_{0}$ is a solution of

$$
\left\{\begin{array}{l}
(\Delta-V) u_{0}=V w \quad \text { in } \Omega, \\
\operatorname{Tr} u_{0}=g \in B_{s}^{p, p}(\partial \Omega), \\
u_{0} \in L_{s+\frac{1}{p}}^{p}(\Omega) .
\end{array}\right.
$$

Now, $w \in L^{n p /(n-1-s p)}(\Omega)$ by standard embedding results and, further, $V w \in L^{q}(\Omega)$ by Hölder's inequality (note that (2.3) guarantees that $q>1)$. This justifies the claim made at the beginning of the paragraph.

Assuming next that $f \in L^{q}(\Omega)$, it follows that $\left\|f^{ \pm}\right\|_{L^{q}(\Omega)} \leq\|f\|_{L^{q}(\Omega)}$. Consider an approximating sequence $V_{j} \rightarrow V$ in $L^{n / 2}(\Omega)$, so that $V_{j} \geq 0$, 
$V_{j} \in L^{\infty}(\Omega)$, for each $j$, and let $u_{j}^{ \pm}$be the unique solution of

$$
\left\{\begin{array}{l}
\left(\Delta-V_{j}\right) u_{j}^{ \pm}=-f^{\mp} \in L^{q}(\Omega), \\
\operatorname{Tr} u_{j}^{ \pm}=g^{ \pm} \in B_{s}^{p, p}(\partial \Omega), \\
u_{j}^{ \pm} \in L_{s+\frac{1}{p}}^{p}(\Omega) .
\end{array}\right.
$$

As before, we get that $\left\|u_{j}^{ \pm}\right\|_{L^{n p /(n-1-s p)}(\Omega)} \leq\left\|u_{0}^{ \pm}\right\|_{L^{n p /(n-1-s p)}(\Omega)}$, where $u_{0}^{ \pm}$solves (2.86) with $V_{0}:=0$. From this and the decomposition $u_{j}=$ $u_{j}^{+}-u_{j}^{-}$, we conclude that there exists some constant $C>0$ independent of $V$ such that

$$
\left\|u_{j}\right\|_{L^{n p /(n-1-s p)}(\Omega)} \leq C\left(\|f\|_{L^{q}(\Omega)}+\|g\|_{B_{s}^{p, p}(\Omega)}\right) .
$$

We now make the claim that $\left(u_{j}\right)_{j \in \mathbb{N}}$ is a Cauchy sequence in $L^{n p /(n-1-s p)}(\Omega)$. To see this, for $j, k \in \mathbb{N}$, we note that $w:=u_{j}-u_{k}$ solves the problem

$$
\left(\Delta-V_{j}\right) w=\left(V_{j}-V_{k}\right) u_{k} \in L^{q}(\Omega), \quad w \in L_{s+\frac{1}{p}, 0}^{p}(\Omega) .
$$

Hence, by virtue of (2.87), we have

$$
\begin{aligned}
\left\|u_{j}-u_{k}\right\|_{L^{n p /(n-1-s p)}(\Omega)} & \leq C\left\|\left(V_{j}-V_{k}\right) u_{k}\right\|_{L^{q}(\Omega)} \\
& \leq C\left\|V_{j}-V_{k}\right\|_{L^{n / 2}(\Omega)}\left\|u_{k}\right\|_{L^{n p /(n-1-s p)}(\Omega)} \\
& \leq C\left\|V_{j}-V_{k}\right\|_{L^{n / 2}(\Omega)}\left(\|f\|_{L^{q}(\Omega)}+\|g\|_{B_{s}^{p, p}(\Omega)}\right) .
\end{aligned}
$$

From this, our claim follows.

In order to continue, let $u$ be the limit of the sequence $\left(u_{j}\right)_{j \in \mathbb{N}}$ in $L^{n p /(n-1-s p)}(\Omega)$. We now intend to show that $u$ solves (2.1). Denote by $u=T_{g}(f)$ the solution operator of the Poisson problem (2.1) corresponding to $V:=0$. In particular, $u_{j}=T_{g}\left(f+V_{j} u_{j}\right)$. Since $V_{j} u_{j} \rightarrow V u$ in $L^{q}(\Omega) \hookrightarrow L_{s+1 / p-2}^{p}(\Omega)$, we may conclude that $u_{j}=T_{g}\left(f+V_{j} u_{j}\right) \rightarrow$ $T_{g}(f+V u)$ in $L_{s+1 / p}^{p}(\Omega)$. From this we see that $u=T_{g}(f+V u)$, i.e., $u$ solves (2.1).

To finish the proof of the theorem we need to show that the solution we have just constructed is unique. By linearity, this comes down to proving that the operator

$$
\Delta-V: L_{s+\frac{1}{p}, 0}^{p}(\Omega) \longrightarrow L_{s+\frac{1}{p}-2}^{p}(\Omega)=\left(L_{2-s-\frac{1}{p}}^{p^{\prime}}(\Omega)\right)^{*}, \quad \frac{1}{p}+\frac{1}{p^{\prime}}=1,
$$

is one-to-one. From what we have proved so far, this operator is onto so, given the invariance of the conditions (2.2)-(2.3) to the transformation $(s, 1 / p) \mapsto(1-s, 1-1 / p)$, the desired result follows by duality. 
Parenthetically, let us note that we could have reached the same conclusion using the ontoness of (2.90) plus the fact that, much as in (2.28), the operator (2.90) is Fredholm with index zero.

\section{Nonlinearities with polynomial growth}

In this section we study the semilinear version of (2.4) in the case when the nonlinearity is allowed to have superlinear growth. In order to state our first result, recall that a function $a(x, u)$ is called Carathéodory if it is measurable in $x$ and continuous in $u$.

Theorem 3.1. Assume that $\Omega \subset M$ is a connected Lipschitz domain in $M$, where $\operatorname{dim} M=n \geq 4$. Also, suppose that $a: \Omega \times \mathbb{R} \rightarrow \mathbb{R}$ is a Carathéodory function such that

$$
0 \leq a(x, u) \leq k_{1}(x)+k_{2}(x)|u|^{m},
$$

where $0 \leq k_{j} \in L^{q_{j}}(\Omega)$ with $q_{j} \geq 1, j=1,2$.

For $1<p<\infty, 0<s<1$, consider the following semilinear Poisson problem with Dirichlet boundary condition:

$$
\begin{aligned}
\Delta u-a(x, u) u & =f \in L_{s+1 / p-2}^{p}(\Omega), \\
\operatorname{Tr} u & =g \in B_{s}^{p, p}(\partial \Omega), \quad u \in L_{s+1 / p}^{p}(\Omega) .
\end{aligned}
$$

Then there exists $\varepsilon=\varepsilon(\Omega)>0$ such that the problem (3.2) has at least one solution provided the following is true: The pair $(p, s)$ satisfies at least one of the conditions in $(2.2), s p<n-1$, and

$$
\begin{aligned}
& 0 \leq \frac{1}{q_{1}} \leq \frac{2}{n} \text { and } \frac{1}{q_{1}}<1-\frac{n-1-s p}{n p} \\
& 0 \leq m<\frac{n p}{n-1-s p}\left(\min \left\{\frac{2}{n}, 1-\frac{n-1-s p}{n p}\right\}-\frac{1}{q_{2}}\right) .
\end{aligned}
$$

For $s p \geq n-1$ the number $m$ could be taken arbitrarily large as long as $q_{1}, q_{2}>\left(\frac{2}{n}+\frac{n-1}{n p}-\frac{s}{n}\right)^{-1}$. Moreover,

$$
\begin{aligned}
& \forall M>0 \quad \exists K>0 \\
& \text { so that }\|f\|_{L_{s+1 / p-2}^{p}(\Omega)}+\|g\|_{B_{s}^{p, p}(\partial \Omega)} \leq M \Longrightarrow\|u\|_{L_{s+1 / p}^{p}(\Omega)} \leq K .
\end{aligned}
$$

If $s p \geq 1$ the solution $u$ satisfies the estimate

$$
\|u\|_{L^{n p /(n-1-s p)}(\Omega)} \leq C\left(\|f\|_{L_{s+1 / p-2}^{p}(\Omega)}+\|g\|_{B_{s}^{p, p}(\partial \Omega)}\right)
$$

for some $C=C(\partial \Omega, s, p, a)>0$. 
If, in addition, the nonlinearity $b(x, u):=a(x, u) u$ satisfies

$$
0 \leq \frac{\partial}{\partial u} b(x, u) \leq \tilde{k}_{1}(x)+\tilde{k}_{2}(x)|u|^{m},
$$

with $0 \leq \tilde{k}_{j} \in L^{q_{j}}(\Omega), j=1,2, q_{j}$ 's and $m$ as before, then the solution $u$ of the boundary problem (3.2) is also unique.

Analogous results are valid in dimensions $n=2$ and $n=3$. If $\operatorname{dim} M=n=3$ the results stated above remain true without change, provided $s p \geq 1 / 2$ and $p(1+s) \geq 2$. Otherwise

$$
0 \leq m<\frac{3 p}{2-s p}\left(\frac{1}{\sigma}-\frac{1}{q_{2}}\right), \quad 0 \leq \frac{1}{q_{1}}<1-\frac{2-s p}{3 p},
$$

will suffice, where

$$
\sigma:= \begin{cases}\frac{3}{2}+\frac{1-2 s p}{2(2-s p)}, & \text { if } 2 s p<1 \text { and } p(3+s) \geq 5, \\ \frac{3}{2}\left(1+\frac{2-p(1+s)}{2-s p}\right), & \text { if } p(1+s)<2 \text { and } p(3+s)<5 .\end{cases}
$$

If $n=2$ the previous results are valid provided (2.10) is used in place of (2.2), and

$$
\begin{array}{lll}
q_{1}>2, & 0 \leq m<\frac{p\left(q_{2}-2\right)}{2 q_{2}(1-s p)} & \text { for } s p<1, \\
q_{1}, q_{2}>\left(1-\left|\frac{1}{2 p}-\frac{s}{2}\right|\right)^{-1}, & m \geq 0, & \text { for } s p \geq 1 .
\end{array}
$$

By way of contrast, $1<p<\infty, 0<s<1$, will do in all dimensions when $\partial \Omega \in C^{1}$.

A few comments are in order here.

(i) As far as the range of validity (described by means of (2.2), (2.10)) is concerned, our theorem is in the nature of best possible. This is because the aforementioned range is optimal for the associated linear problems.

(ii) In some instances $m$ in (3.4) can be allowed to attain the value $\frac{n p}{n-1-s p}\left(\frac{2}{n}-\frac{1}{q_{2}}\right)$ and the statement of the theorem remains true. In order to avoid further complications we decided not to include this case in the theorem given above. Interested reader can analyze this boundary case by techniques outlined in the proof that follows.

(iii) Similar results are valid at the level of $2^{\text {nd }}$-order, formally selfadjoint, non-positive, strongly elliptic systems, at least when $n=2$ or $n=3$. 
(iv) These results can be extended to allow other types of nonlinear terms. We discuss the case of sub-linear nonlinearities in Section 5, while in Section 6 we consider other classes of function spaces.

Proof of Theorem 3.1: Assume first that $s p<n-1$. The idea is to consider the map

$$
T: L^{n p /(n-1-s p)}(\Omega) \longrightarrow L^{n p /(n-1-s p)}(\Omega)
$$

defined as follows. For a fixed $g \in B_{s}^{p, p}(\partial \Omega)$ and $f \in L_{s+1 / p-2}^{p}(\Omega)$ we take $v=T u$ to be the solution of the linear Poisson problem

$$
\Delta v-a(x, u) v=f \text { in } \Omega, \quad \operatorname{Tr} v=g, \quad v \in L_{s+1 / p}^{p}(\Omega) .
$$

The first order of business is to show that $T$ is well defined. Sufficient conditions have been discussed in Theorem 2.1 in terms of the integrability properties of $V(x):=a(x, u(x))$. In this regard, we note that

$$
\begin{aligned}
& \frac{1}{r} \geq \max \left\{\frac{m}{\tau}+\frac{1}{q_{2}}, \frac{1}{q_{1}}\right\} \Longrightarrow a(., u(.)) \in L^{r}(\Omega) \\
& \text { and }\|a(., u(.))\|_{L^{r}(\Omega)} \leq C\left(\left\|k_{1}\right\|_{L^{q_{1}}(\Omega)}+\left\|k_{2}\right\|_{L^{q_{2}}(\Omega)}\|u\|_{L^{\tau}(\Omega)}^{m}\right),
\end{aligned}
$$

with $\tau:=n p /(n-1-s p)$, as is immediate from (3.1). Next, suppose that $r$ has been selected as in the first line of (3.13) and assume that $r \geq n / 2$. The intention is to employ the estimates (2.6), (2.8) in order to deduce that

$$
\text { (3.14) }\|v\|_{L^{\tau}(\Omega)} \leq C\left(1+\|a(., u(.))\|_{L^{r}(\Omega)}\right)^{\theta}\left(\|f\|_{L_{s+1 / p-2}^{p}(\Omega)}+\|g\|_{B_{s}^{p, p}(\partial \Omega)}\right)
$$

with $C>0$ independent of $u$, and $\theta=\theta(n, s, p, r) \in[0,1)$. Here $\theta:=0$ for $s p \geq 1$, and is given by (2.9) when $0<s<\frac{1}{p}<\frac{s}{n-1}+\frac{n(r-1)}{r(n-1)}$. Note that this last estimate entails $1 / r<1-1 / \tau$, which can be viewed as a necessary condition for $(s, 1 / p)$ to belong to the region where estimates for $\theta$ are available (cf. (i)-(iv) in Lemma 2.9).

In summary, carrying out the step outlined in the previous paragraph requires that

$$
\max \left\{\frac{m}{\tau}+\frac{1}{q_{2}}, \frac{1}{q_{1}}\right\} \leq \frac{1}{r} \leq \frac{2}{n} \text { and } \frac{1}{r}<1-\frac{1}{\tau} .
$$

Clearly, the possibility of choosing $r$ as in (3.15) hinges on whether

$$
\max \left\{\frac{m}{\tau}+\frac{1}{q_{2}}, \frac{1}{q_{1}}\right\} \leq \frac{2}{n} \text { and } \max \left\{\frac{m}{\tau}+\frac{1}{q_{2}}, \frac{1}{q_{1}}\right\}<1-\frac{1}{\tau},
$$

a condition which is equivalent to (3.3)-(3.4). 
Assuming that this is the case and choosing $r$ as in (3.15), at this stage we would like to be able to pick some $R>0$ so that $T$ maps the ball $\left\{u \in L^{\tau}(\Omega) ;\|u\|_{L^{\tau}(\Omega)} \leq R\right\}$ into itself, i.e.

$$
u \in L^{\tau}(\Omega), \quad\|u\|_{L^{\tau}(\Omega)} \leq R \Longrightarrow\|T u\|_{L^{\tau}(\Omega)} \leq R .
$$

Another look at (3.13)-(3.14) reveals that matters can always be arranged to that effect (by selecting $R$ large enough) granted that

$$
m \theta<1 \text {. }
$$

Checking (3.18) amounts to analyzing several cases.

(i) If $s p \geq 1$ then this is trivially satisfied, since $\theta=0$ in this case.

(ii) If $\theta$ is as in the first line of (2.9), we write (3.18) in a way which emphasizes $1 / r$, i.e. $\frac{1}{r}<1-\frac{m}{2}\left(\frac{1}{p}-s\right)$. In order to be able to select $r$ as in (3.15) which also satisfies this latest condition we therefore need

$$
\max \left\{\frac{m}{\tau}+\frac{1}{q_{2}}, \frac{1}{q_{1}}\right\}<1-\frac{m}{2}\left(\frac{1}{p}-s\right),
$$

or, equivalently,

(3.20) $\quad 0 \leq m$

$$
<\min \left\{\left(1-\frac{1}{q_{2}}\right)\left(\frac{1}{\tau}+\frac{1}{2}\left(\frac{1}{p}-s\right)\right)^{-1}, 2\left(1-\frac{1}{q_{1}}\right)\left(\frac{1}{p}-s\right)^{-1}\right\} .
$$

We now make the claim that (3.4) implies (3.20) whenever $n \geq 4$, or if $n=3$ provided $2 s p \geq 1$. With this aim in mind, it is sufficient to verify that

$$
\begin{aligned}
& \left(\frac{2}{n}-\frac{1}{q_{2}}\right) \tau<\left(1-\frac{1}{q_{2}}\right)\left(\frac{1}{\tau}+\frac{1}{2}\left(\frac{1}{p}-s\right)\right)^{-1}, \\
& \left(\frac{2}{n}-\frac{1}{q_{2}}\right) \tau<2\left(1-\frac{1}{q_{1}}\right)\left(\frac{1}{p}-s\right)^{-1},
\end{aligned}
$$

under the hypotheses just mentioned, a task to which we now turn. Given the linear nature of both sides of (3.21) in the parameter $1 / q_{2} \in$ $(0,2 / n]$ and the fact that $(3.21)$ holds when $1 / q_{2}=2 / n$, it suffices to check (3.21) when $1 / q_{2}=0$. After some algebra, this limiting case eventually comes down to verifying that

$$
n^{2}-4 n+2 \geq-2 s p,
$$

which clearly holds if $n \geq 4$, or if $n=3$ and $2 s p \geq 1$. Verifying (3.22) is done in a similar fashion; once again, matters are reduced precisely 
to (3.23). Finally, when $n=3$ and $2 s p \geq 1, \frac{1}{p}-\frac{s}{2} \leq \frac{1}{2}$, then necessarily $\theta$ is as in the first line of (2.9) and, from what we have just proved, (3.18) holds. This proves the claim made at the beginning of the paragraph.

(iii) Consider next the case when $\theta$ is as in the second line of (2.9). Note that a necessary condition for this to happen is $\frac{1}{p}-\frac{s}{n-1}>\frac{n-2}{n-1}$ (as a simple geometrical argument shows) or, equivalently,

$$
1-\frac{2}{n}<\frac{1}{\tau}
$$

Then, in the case we are considering, $m \theta<1$ becomes equivalent to the estimate $\frac{1}{r}<\frac{2}{n}-m\left(\frac{1}{\tau}-\frac{n-2}{n}\right)$. In turn, having this compatible with (3.15) requires

(3.25) $\quad 0 \leq m$

$$
<\min \left\{\left(\frac{2}{n}-\frac{1}{q_{2}}\right)\left(\frac{2}{\tau}-\frac{n-2}{n}\right)^{-1},\left(\frac{2}{n}-\frac{1}{q_{1}}\right)\left(\frac{1}{\tau}-\frac{n-2}{n}\right)^{-1}\right\} .
$$

We now make the claim that (3.15) and (3.24) always imply (3.25) when $n \geq 4$. Clearly, this will follows as soon as we prove that, for $n \geq 4$,

$$
\begin{aligned}
& \tau\left(1-\frac{1}{\tau}-\frac{1}{q_{2}}\right) \leq\left(\frac{2}{n}-\frac{1}{q_{2}}\right)\left(\frac{2}{\tau}-\frac{n-2}{n}\right)^{-1}, \\
& \tau\left(1-\frac{1}{\tau}-\frac{1}{q_{2}}\right) \leq\left(\frac{2}{n}-\frac{1}{q_{1}}\right)\left(\frac{1}{\tau}-\frac{n-2}{n}\right)^{-1} .
\end{aligned}
$$

Since (3.26) reduces to (3.24) if $\frac{1}{q_{2}}=\frac{2}{n}$, it is enough to consider the case $\frac{1}{q_{2}}=0$ only, when (3.26) reduces to

$$
\left(\frac{1}{\tau}-\frac{1}{2}\right)\left(\frac{1}{\tau}-\frac{n-2}{n}\right) \geq 0 .
$$

On the other hand, (3.24) implies (3.28) as long as $n \geq 4$. As for (3.27), it suffices to check the situation when $\frac{1}{q_{1}}=1-\frac{1}{\tau}$ and $\frac{1}{q_{2}}=\frac{2}{n}$. This, however, is clearly true for each $n \geq 3$, thanks to (3.24). The claim made at the beginning of the current paragraph is therefore justified.

Summarizing, at this point we have proved that, granted (3.16), matters can be arranged so that (3.18) holds unconditionally when $n \geq 4$, or if $n=3$ provided $2 s p \geq 1$ and $p(1+s) \geq 2$.

We now discuss a variant of the above reasoning which will eventually allow us to get manageable conditions on $m, q_{1}, q_{2}$ in the remaining cases as well. Suppose for a moment that, given $r$ so that 
$\frac{1}{r} \geq \max \left\{\frac{m}{\tau}+\frac{1}{q_{2}}, \frac{1}{q_{1}}\right\}$, we demand that $r$ is sufficiently large instead of the usual condition $r \geq n / 2$. Say, $r>\sigma$, where $\sigma \geq n / 2$ is a parameter to be specified later. Enforcing this additional restriction requires

$$
\max \left\{\frac{m}{\tau}+\frac{1}{q_{2}}, \frac{1}{q_{1}}\right\}<\min \left\{\frac{1}{\sigma}, 1-\frac{1}{\tau}\right\}
$$

in lieu of (3.16). Once again, the immediate goal is to ensure that, granted (3.29), the estimate (3.18) holds. With this in mind, in place of (3.21)-(3.22) and (3.26)-(3.27) it suffices to check that

$$
\begin{aligned}
& \left(\frac{1}{\sigma}-\frac{1}{q_{2}}\right) \tau<\left(1-\frac{1}{q_{2}}\right)\left(\frac{1}{\tau}+\frac{1}{2}\left(\frac{1}{p}-s\right)\right)^{-1}, \\
& \left(\frac{1}{\sigma}-\frac{1}{q_{2}}\right) \tau<2\left(1-\frac{1}{q_{1}}\right)\left(\frac{1}{p}-s\right)^{-1},
\end{aligned}
$$

and

$$
\begin{aligned}
& \left(\frac{1}{\sigma}-\frac{1}{q_{2}}\right) \tau \leq\left(\frac{2}{n}-\frac{1}{q_{2}}\right)\left(\frac{2}{\tau}-\frac{n-2}{n}\right)^{-1}, \\
& \left(\frac{1}{\sigma}-\frac{1}{q_{2}}\right) \tau \leq\left(\frac{2}{n}-\frac{1}{q_{1}}\right)\left(\frac{1}{\tau}-\frac{n-2}{n}\right)^{-1},
\end{aligned}
$$

respectively, where $0<\frac{1}{q_{2}}<\frac{1}{\sigma}$ and $0<\frac{1}{q_{1}}<\min \left\{\frac{1}{\sigma}, 1-\frac{1}{\tau}\right\}$. Much as before, it suffices to check (3.30)-(3.31) only for suitable endpoint values of $1 / q_{1}, 1 / q_{2}$; we obtain:

$$
\begin{aligned}
& \sigma \geq 1+\frac{\tau}{2}\left(\frac{1}{p}-s\right) \Longrightarrow(3.30) \text { holds; } \\
& \sigma \geq n-\frac{\tau(n-2)}{2} \Longrightarrow \text { (3.31) holds. }
\end{aligned}
$$

It is now easy to see that implementing the strategy just outlined for $n=3$ and $n=2$ leads to the choices (3.8), (3.9), (3.10). Finally, under the same hypotheses on the numerical parameters as in the Lipschitz case, $\partial \Omega \in C^{1}$ can also be allowed since the first inequality in (2.3) is contained in (3.15).

Next, we make the claim that $T$ in (3.11) is continuous and compact. Continuity follows from Theorem 2.1 and the fact that the map $u \mapsto$ $a(., u()$.$) is continuous from L^{\tau}(\Omega)$ into $L^{r}(\Omega)$ (since $a$ is Carathéodory, the latter fact is a simple consequence of (3.1), (3.4) and Egoroff's theorem). 
To show compactness, let $\left(u_{i}\right)_{i \geq 1}$ be any bounded sequence in $L^{\tau}(\Omega)$. From (3.14) we get that $v_{i}:=T u_{i}$ are also uniformly bounded in $L^{\tau}(\Omega)$. Hence, introducing

$$
h_{i}:=a\left(x, u_{i}(x)\right) v_{i}(x), \quad x \in \Omega, i=1,2, \ldots,
$$

we learn from Lemma 2.2 that $\left\{h_{i}\right\}_{i}$ is relatively compact in $L_{s+1 / p-2}^{p}(\Omega)$. Thus, by eventually passing to a subsequence (a fact which our notation does not actually reflect), we can assume that $h_{i}$ converges to some $h^{*}$ in $L_{s+1 / p-2}^{p}(\Omega)$. In particular, since

$$
\Delta v_{i}=f+h_{i} \text { in } \Omega, \quad \operatorname{Tr} v_{i}=g, \quad v_{i} \in L_{s+1 / p}^{p}(\Omega), \quad i=1,2, \ldots,
$$

from the well posedness of the linear problem (cf. Theorem 2.1) we may conclude that $v_{i}$ converges to some $v^{*}$ in $L_{s+1 / p}^{p}(\Omega)$. Consequently, $v_{i} \rightarrow$ $v^{*}$ in $L^{\tau}(\Omega)$ and this finishes the proof of the fact that $T$ is compact.

On account of (3.17), Schauder's fixed-point theorem finally yields that $T$ has a fixed point $u$. This, in turn, solves (3.2) and obeys (3.5).

There remains uniqueness which we tackle next. The starting point is the observation that if $u_{1}, u_{2}$ are two solutions of (3.2), then $v:=u_{1}-u_{2}$ satisfies

$$
(\Delta-\tilde{V}) v=0 \text { in } \Omega, \quad v \in L_{s+1 / p, 0}^{p}(\Omega),
$$

where $\tilde{V}$ is given by

$$
\tilde{V}(x):=\int_{0}^{1}\left(\partial_{u} b\right)\left(x, u_{2}(x)+t\left(u_{1}(x)-u_{2}(x)\right)\right) d t .
$$

Note that, due to our current assumptions,

$$
0 \leq \tilde{V}(x) \leq \tilde{k}_{1}(x)+\tilde{k}_{2}(x) \max \left\{\left|u_{1}(x)\right|^{m},\left|u_{2}(x)\right|^{m}\right\} .
$$

In particular, $\tilde{V} \in L^{r}(\Omega)$ for some $r \geq n / 2$, so that $v=0$ (i.e. $u_{1}=u_{2}$ ) from the uniqueness part in Theorem 2.1. This finishes the analysis of the case $s p<n-1$.

The case when $s p \geq n-1$ is treated in a similar fashion and this completes the proof of the theorem.

Our next result shows that, in the previous theorem, the polynomial growth condition (3.1) can be further relaxed to an exponential type bound in the case $s p \geq n-1$. Specifically, we have the following. 
Theorem 3.2. Let $a: \Omega \times \mathbb{R} \rightarrow \mathbb{R}$ be a Carathéodory function and for $1<p<\infty, 0<s<1$, consider the following semilinear Poisson problem with Dirichlet boundary condition:

$$
\begin{aligned}
\Delta u-a(x, u) u & =f \in L_{s+1 / p-2}^{p}(\Omega), \\
\operatorname{Tr} u & =g \in B_{s}^{p, p}(\partial \Omega), \quad u \in L_{s+1 / p}^{p}(\Omega) .
\end{aligned}
$$

Then there exists $\varepsilon=\varepsilon(\Omega)>0$ with the following significance. Suppose that the pair $(p, s)$ satisfies at least one of the conditions in (2.2) and that either

(a) $s p=n-1$ and for each $A>0$ there exists $k \in L^{t}(\Omega), t>n / 2$, such that

$$
0 \leq a(x, u) \leq k(x) \exp \left(A|u|^{\gamma}\right), \quad \text { with } \quad \gamma:=\gamma(p)=p /(p-1),
$$

or

(b) $s p>n-1$ and

(3.40) $0 \leq a(x, u), \quad \forall M>0 \quad \sup _{u \in[-M, M]}|a(x, u)| \in L^{t}(\Omega)$ for some $t>\sigma$, where $\sigma:=\left(\frac{2}{n}+\frac{n-1}{n p}-\frac{s}{n}\right)^{-1}$.

Then the boundary problem (3.38) has at least one solution u. Moreover, matters can be arranged so that

$$
\|u\|_{L^{\tau}(\Omega)} \leq C \tau^{1-1 / p}\left(\|f\|_{L_{s+1 / p-2}^{p}(\Omega)}+\|g\|_{B_{s}^{p, p}(\partial \Omega)}\right)
$$

for some $C=C(\partial \Omega, s, p, a)>0$ and any $\tau \in[2, \infty)$, provided (a) holds. If (b) holds we have that

$$
\|u\|_{L^{\infty}(\Omega)} \leq C\left(\|f\|_{L_{s+1 / p-2}^{p}(\Omega)}+\|g\|_{B_{s}^{p, p}(\partial \Omega)}\right)
$$

for some $C=C(\partial \Omega, s, p, a)>0$. Furthermore, in either case

$$
\begin{aligned}
& \forall M>0 \quad \exists K>0 \\
& \text { so that }\|f\|_{L_{s+1 / p-2}^{p}(\Omega)}+\|g\|_{B_{s}^{p, p}(\partial \Omega)} \leq M \Longrightarrow\|u\|_{L_{s+1 / p}^{p}(\Omega)} \leq K .
\end{aligned}
$$

If, in addition, the nonlinearity $b(x, u):=a(x, u) u$ satisfies one of the following two conditions: 
(a) $s p=n-1$ and for each $A>0$ there exists $\tilde{k} \in L^{t}(\Omega), t>n / 2$, such that

(3.44) $0 \leq \frac{\partial}{\partial u} b(x, u) \leq \tilde{k}(x) \exp \left(A|u|^{\gamma}\right), \quad$ with $\quad 0 \leq \gamma=\gamma(p)=p /(p-1)$,

(b) $s p>n-1$ and

$$
\begin{aligned}
0 \leq & \frac{\partial}{\partial u} b(x, u), \\
& \forall M>0 \quad \sup _{u \in[-M, M]}\left|\frac{\partial}{\partial u} b(x, u)\right| \in L^{t}(\Omega) \text { for some } t>\sigma,
\end{aligned}
$$

then the solution $u$ of the boundary problem (3.38) is also unique.

Finally, analogous results are valid in dimension $n=2$, provided (2.10) is used in place of (2.2). Moreover, $1<p<\infty, 0<s<1$, $s p \geq n-1$, will do in all dimensions when $\partial \Omega \in C^{1}$.

Proof: Consider first the somewhat simpler case $s p>n-1$. It follows from Theorem 2.1 that any solution to the equation (2.4) satisfies

$$
\|u\|_{L^{\infty}(\Omega)} \leq C\left(\|f\|_{L_{s+\frac{1}{p}-2}^{p}(\Omega)}+\|g\|_{B_{s}^{p, p}(\partial \Omega)}\right),
$$

with $C>0$ independent of $V \geq 0$. Introduce $N:=C\left(\|f\|_{L_{s+\frac{1}{p}-2}^{p}(\Omega)}+\right.$ $\left.\|g\|_{B_{s}^{p, p}(\partial \Omega)}\right)$, then define

$$
\psi_{N}(u):= \begin{cases}u, & \text { for }|u| \leq N \\ N, & \text { for } u>N \\ -N, & \text { for } u<-N\end{cases}
$$

and, finally, consider the Poisson problem:

$$
\begin{aligned}
\Delta u-a\left(x, \psi_{N}(u)\right) u & =f \in L_{s+1 / p-2}^{p}(\Omega), \\
\operatorname{Tr} u & =g \in B_{s}^{p, p}(\partial \Omega), \quad u \in L_{s+1 / p}^{p}(\Omega) .
\end{aligned}
$$

The problem (3.48) satisfies all the assumptions in Theorem 3.1 and, hence, has at least one solution $u$. Moreover, matters can be arranged so that the solution also satisfies the estimate (3.46). This forces $a(x, u(x))=a\left(x, \psi_{N}(u(x))\right)$, thus $u$ solves (3.38) as well. The proof of the uniqueness part parallels its counterpart in Theorem 3.1. 
Next, we turn our attention to the more interesting case $s p=n-1$. To begin with, define the vector space $\mathcal{X}^{p}$ by setting

$$
\mathcal{X}^{p}=\left\{h: \Omega \longrightarrow \mathbb{R} ; \sup _{\tau \geq 2} \tau^{-1+1 / p}\|h\|_{L^{\tau}(\Omega)}<\infty\right\},
$$

and equip it with the norm

$$
\|h\|_{\mathcal{X}^{p}}:=\sup _{\tau \geq 2} \tau^{-1+1 / p}\|h\|_{L^{\tau}(\Omega)} .
$$

It is not very difficult to check that $\mathcal{X}^{p}$ is indeed a Banach space. An essential property enjoyed by functions in $\mathcal{X}^{p}$ is that there exists $\kappa=$ $\kappa(M, \Omega)>0$ such that

$$
h \in \mathcal{X}^{p}, \quad \alpha:=\frac{\kappa}{\|h\|_{\mathcal{X}^{p}}^{p /(p-1)}} \Longrightarrow \exp \left(\alpha|h|^{p /(p-1)}\right) \in L^{1}(\Omega) .
$$

The proof of this fact can be carried out exactly as the proof of Trudinger's inequality; cf. [36, Chapter 13] for more details. Taking another look at the estimate (2.7), we learn that the solution $u$ to the equation (2.4) belongs to $\mathcal{X}^{p}$ and there exists $C>0$ independent of $V$ such that

$$
\|u\|_{\mathcal{X}^{p}} \leq C\left(\|f\|_{L_{s+\frac{1}{p}-2}^{p}(\Omega)}+\|g\|_{B_{s}^{p, p}(\partial \Omega)}\right) .
$$

To proceed from here, consider first the case when $f \in L^{\infty}(\Omega)$. Denote by $u_{V}^{( \pm)}$the solution to the boundary problem:

$$
(\Delta-V) u_{V}^{( \pm)}=f^{\mp} \text { in } \Omega, \quad \operatorname{Tr} u_{V}^{( \pm)}=g^{ \pm} .
$$

In this situation, relying on Lemma 2.3 we infer that $0 \leq u_{V}^{( \pm)} \leq u_{0}^{( \pm)}$, hence there exists $h=u_{0}^{(+)}+u_{0}^{(-)} \in \mathcal{X}^{p}$ such that for any $V \geq 0$ the solution $u_{V}$ of (2.4) satisfies

$$
\left|u_{V}(x)\right| \leq h(x), \quad \text { for any } x \in \Omega .
$$

Let us now define

$$
\mathcal{O}:=\left\{u \in L^{1}(\Omega) ;|u| \leq h\right\},
$$

so that, clearly, $\mathcal{O} \subset \mathcal{X}^{p}$. Another simple yet useful observation is that for any $\tau \in[2, \infty)$ the set $\mathcal{O}$ is closed and convex in $L^{\tau}(\Omega)$. Finally, consider the operator $T: \mathcal{O} \rightarrow \mathcal{O}$ defined by agreeing that $v:=T u$ is the (unique) solution of

$$
\Delta v-a(x, u) v=f \text { in } \Omega, \quad \operatorname{Tr} v=g, \quad v \in L_{s+1 / p}^{p}(\Omega) .
$$


In order to see that $v$ is well defined, it is sufficient to observe that for any $u \in \mathcal{O}$ we have $a(x, u(x)) \in L^{r}(\Omega)$ for some $r>n / 2$. This, however, is a consequence of

$$
0 \leq a(x, u) \leq k(x) \exp \left(A|u|^{\gamma}\right) \leq k(x) \exp \left(A|h|^{\gamma}\right)
$$

Indeed, since $h \in \mathcal{X}^{p}$, one can select $A$ small enough so that $\exp \left(A|h|^{\gamma}\right) \in$ $L^{q}(\Omega)$. Thus, the desired conclusion follows by choosing $q$ sufficiently large, so that $1 / q+1 / t<2 / n$. Also $v=T u \in \mathcal{O}$ by (3.54). Finally, $T$ is a continuous and compact map in the $L^{\tau}(\Omega)$ topology for any $\tau \in[2, \infty)$. Continuity follows from the fact that if $u_{i} \rightarrow u$ pointwise on $\Omega$ and all $u_{i} \in \mathcal{O}$, then also $a\left(., u_{i}().\right) \rightarrow a(., u()$.$) pointwise. As (3.57) gives$ a common majorant in $L^{r}(\Omega)$ (for some fixed $r>n / 2$ ), we see that $a\left(., u_{i}().\right) \rightarrow a(., u()$.$) in L^{r}(\Omega)$. With this at hand, Theorem 2.1 readily finishes the proof of the continuity of $T$. The proof of the compactness of $T$ goes exactly as in Theorem 3.1. Hence, by Schauder's fixed-point theorem, the map $T$ has a fixed point $u \in \mathcal{O}$ which, in turn, is the solution of the Poisson problem we seek. Moreover, $u$ satisfies (3.52).

There remains to show how to dispense off the extra hypothesis $f \in$ $L^{\infty}(\Omega)$. The idea is to approximate $f$ in $L_{s+1 / p-2}^{p}(\Omega)$ by a sequence of functions $f_{j} \in L^{\infty}(\Omega)$. Granted what we have proved so far, at each step $j$, we then solve

$$
\Delta u_{j}-a\left(x, u_{j}\right) u_{j}=f_{j} \text { in } \Omega, \quad \operatorname{Tr} u_{j}=g, \quad u_{j} \in L_{s+1 / p}^{p}(\Omega) .
$$

By $(3.52),\left(u_{j}\right)_{j}$ is a bounded sequence in $\mathcal{X}^{p}$. Going further, this entails that $V_{j}(x):=a\left(x, u_{j}(x)\right)$ are uniformly bounded in $L^{r}(\Omega)$, for some $r>n / 2$. Since we have that $u_{j}=T_{g}\left(f+V_{j} u_{j}\right)$ (here $T_{g}$ has the same meaning as at the end of Section 2), an application of Lemma 2.2 shows that the sequence $\left(u_{j}\right)_{j \geq 1}$ is bounded in $L_{s+1 / p}^{p}(\Omega)$ and that there exists a subsequence which converges to some $u$ in the $L_{s+1 / p}^{p}(\Omega)$ norm. In turn, this readily yields that $u$ solves (3.38), as desired. Finally, the proof of uniqueness is essentially the same as before.

\section{Neumann boundary conditions}

Building on the sharp linear theory from $[\mathbf{9}],[\mathbf{3 0}]$, in this section we analyze the semilinear Poisson problem with nonlinear Neumann boundary conditions. 
Theorem 4.1. Let $\Omega \subset M$ be an arbitrary Lipschitz domain with outward unit conormal $\nu \in T^{*} M$. Also, fix a nonnegative function a $(x, u) \in$ $L^{\infty}(\Omega \times \mathbb{R})$ and consider $L=\Delta-V$, where $V \in L^{r}(\Omega)$ for some $r>n$, $V \geq 0$ and $V>0$ on a set of positive measure in $\Omega$. Then there exists $\varepsilon=\varepsilon(\Omega, a) \in(0,1]$ with the following significance. If $s \in(0,1)$, $p \in(1, \infty)$ satisfy either one of the three conditions in $(2.2)$ (or, respectively, (2.10) if $n=2)$ and if $q:=(1-1 / p)^{-1}, \lambda \in \mathbb{R}, 0<\delta<1$, then the Neumann Poisson problem

$$
(N P)\left\{\begin{array}{l}
L u-a(x, u) u=f \in L_{\frac{1}{q}-s-1,0}^{q}(\Omega), \\
\partial_{\nu} u+\lambda|u|^{\delta}=g \in B_{-s}^{q, q}(\partial \Omega), \\
u \in L_{1-s+\frac{1}{q}}^{q}(\Omega)
\end{array}\right.
$$

has at least a solution which satisfies

$$
\begin{aligned}
& \forall M>0 \quad \exists K>0 \\
& \text { so that }\|f\|_{L_{\frac{1}{q}-s-1,0}^{q}(\Omega)}+\|g\|_{B_{-s}^{q, q}(\partial \Omega)} \leq M \Longrightarrow\|u\|_{L_{\frac{1}{q}-s+1}^{q}(\Omega)} \leq K
\end{aligned}
$$

In the case of linear boundary conditions, i.e., when $\lambda=0$, and when the nonlinearity $b(x, u):=a(x, u) u$ also satisfies $0 \leq \partial_{u} b(x, u) \in L^{\infty}(\Omega \times$ $\mathbb{R})$, the solution is also unique.

Furthermore, when $\partial \Omega \in C^{1}$, we can take any $s \in(0,1)$ and $p \in$ $(1, \infty)$

Proof: If $u \in L_{1-s+\frac{1}{q}}^{q}(\Omega)$, then $\operatorname{Tr} u \in B_{1-s}^{q, q}(\partial \Omega)$ and recall from [33] that

$$
|\cdot|^{\delta}: B_{1-s}^{q, q}(\partial \Omega) \longrightarrow B_{(1-s) \delta}^{q / \delta, q / \delta}(\partial \Omega)
$$

is well-defined and bounded. Furthermore, by allowing an arbitrary small defect of smoothness (for the target space), this operator also becomes continuous and compact; cf. [32, Remark 5, p. 377].

Next, with $u$ as above, we let $v:=T_{f, g}(u)$ be the unique solution of the linear Poisson problem

$$
\left\{\begin{array}{l}
L v-a(x, u) v=f \in L_{\frac{1}{q}-s-1,0}^{p}(\Omega), \\
\partial_{\nu} v=g-\lambda|u|^{\delta} \in B_{-s}^{q, q}(\partial \Omega), \\
v \in L_{1-s+\frac{1}{q}}^{q}(\Omega) .
\end{array}\right.
$$


That the latter is well posed, granted the current hypotheses, is guaranteed by the results in $[\mathbf{3 0}]$. We aim at showing that

$$
T_{f, g}: L_{1-s+\frac{1}{q}}^{q}(\Omega) \longrightarrow L_{1-s+\frac{1}{q}}^{q}(\Omega)
$$

is a continuous, compact mapping.

First, let $u_{j} \rightarrow u_{0}$ in $L_{1-s+\frac{1}{q}}^{q}(\Omega)$ and set $v_{j}:=T_{f, g}\left(u_{j}\right), v_{0}:=T_{f, g}\left(u_{0}\right)$. We will show that

$$
v_{j} \longrightarrow v_{0} \quad \text { in } \quad L_{1-s+\frac{1}{q}}^{q}(\Omega) .
$$

Clearly, it suffices to prove the convergence in (4.6) for a subsequence (still denoted $\left.\left(v_{j}\right)_{j \geq 1}\right)$. Since, by the estimates of the linear theory and (4.3), there exists $\bar{C}=C\left(\|a\|_{L^{\infty}}, \Omega, s, q, \lambda\right)>0$ such that

$$
\left\|v_{j}\right\|_{L_{1-s+\frac{1}{q}}^{q}(\Omega)} \leq C\left(\|f\|_{L_{\frac{1}{q}-s-1}^{q}(\Omega)}+\|g\|_{B_{-s}^{q, q}(\partial \Omega)}+\left\|u_{j}\right\|_{L_{\frac{1}{q}-s-1}^{q}}^{\delta}(\Omega)\right),
$$

an easy application of Rellich's selection lemma, in concert with the uniqueness in the linear theory, gives that for each small $\varepsilon>0$ (and after possibly restricting to a subsequence),

$$
v_{j} \longrightarrow v_{0} \quad \text { in } \quad L_{1-s+\frac{1}{q}-\varepsilon}^{q}(\Omega) .
$$

Next, observe that $v_{j}-v_{k} \in L_{1-s+\frac{1}{q}}^{q}(\Omega)$ satisfies

$$
\begin{array}{r}
L\left(v_{j}-v_{k}\right)=a\left(x, u_{j}\right) v_{j}-a\left(x, u_{k}\right) v_{k} \longrightarrow 0 \quad \text { in } L_{1-s+\frac{1}{q}}^{q}(\Omega), \\
\text { and } \partial_{\nu}\left(v_{j}-v_{k}\right) \longrightarrow 0 \text { in } B_{-s}^{q, q}(\Omega) .
\end{array}
$$

Hence, once again by virtue of the estimates in the linear theory,

$$
\left\|v_{j}-v_{k}\right\|_{L_{1-s+\frac{1}{q}}^{q}(\Omega)} \longrightarrow 0 .
$$

Clearly, this and (4.8) yield (4.6), hence the operator in (4.5) is continuous.

Turning attention to the compactness of $T_{f, g}$ in (4.5), assume that $u_{j} \in L_{1-s+\frac{1}{q}}^{q}(\Omega)$ is an arbitrary bounded sequence and set

$$
\begin{aligned}
& v_{j}:=T_{f, g}\left(u_{j}\right), \\
& f_{j}:=f+a\left(x, u_{j}\right) v_{j} \in L_{\frac{1}{q}-s-1,0}^{q}(\Omega), \\
& g_{j}:=g-\lambda\left|u_{j}\right|^{\delta} \in B_{-s}^{q, q}(\partial \Omega) .
\end{aligned}
$$


Since $a\left(x, u_{j}\right) \in L^{\infty}, v_{j}$ is a bounded sequence in $L_{1-s+\frac{1}{q}}^{q}(\Omega)$ (cf. (4.7)) and the embedding

$$
L^{\infty}(\Omega) \cdot L_{1-s+\frac{1}{q}}^{q}(\Omega) \hookrightarrow L_{\frac{1}{q}-s-1,0}^{q}(\Omega)
$$

is compact, there is no loss of generality (cf. also the discussion pertaining to (4.3)) assuming that

$$
\begin{aligned}
& f_{j} \text { converges to some } f_{0} \text { in } L_{\frac{1}{q}-s-1,0}^{q}(\Omega), \\
& \text { and } g_{j} \text { converges to some } g_{0} \text { in } B_{-s}^{q, q}(\partial \Omega) .
\end{aligned}
$$

Granted this and observing that

$$
\left\{\begin{array}{l}
L v_{j}=f_{j} \in L_{\frac{1}{q}-s-1,0}^{q}(\Omega), \\
\partial_{\nu} v_{j}=g_{j} \in B_{-s}^{q, q}(\partial \Omega), \\
v_{j} \in L_{1-s+\frac{1}{q}}^{q}(\Omega),
\end{array}\right.
$$

it follows from the well-posedness of the linear problem that $v_{j} \rightarrow v_{0}$ in $L_{1-s+\frac{1}{q}}^{q}(\Omega)$, where $v_{0}$ is the unique solution of (4.14) with $f_{j}, g_{j}$ replaced, respectively, by $f_{0}$ and $g_{0}$. This proves that the operator in (4.5) is also compact.

Note that if we let $B_{R}$ stand for the closed ball (centered at the origin) of radius $R$ in the space $L_{1-s+\frac{1}{q}}^{q}(\Omega)$ then, by (4.7), the operator $T_{f, g}: B_{R} \rightarrow B_{R}$ is well-defined, continuous and compact, provided $R$ is large enough. At this stage, Schauder's fixed-point theorem applies and takes care of the solvability of the Neumann Poisson problem (4.1), as well as, the accompanying estimate (4.2).

Finally, granted that $\lambda=0$ and the extra condition $0 \leq \partial_{u}[a(x, u) u] \in$ $L^{\infty}(\Omega \times \mathbb{R})$, uniqueness for the problem (4.1) can be readily reduced to its linear version, i.e., when $a(x, u)$ is independent of $u$. The reasoning when $\partial \Omega \in C^{1}$ is similar and this finishes the proof.

\section{Nonlinearities with sublinear growth}

We retain our standard hypotheses on $M$ and $\Omega$ made in Theorem 3.1. As usual, set $L=\Delta-V$. The main result of this section is the following. 
Theorem 5.1. Let $N(x, u)$ be a Carathéodory function such that

$$
|N(x, u)| \leq k_{1}(x)+k_{2}(x)|u(x)|^{\delta}, \quad \text { for some } 0 \leq \delta<1,
$$

where $0 \leq k_{j} \in L^{q_{j}}(\Omega)$ with $q_{j}>n / 2, j=1,2$. Also, assume that $V$ satisfies (2.1). Then, if $s, p$ satisfy either one of the three conditions in (2.2) and if (2.3) holds, the Poisson problem with Dirichlet boundary condition

$$
\begin{aligned}
L u-N(x, u) & =f \in L_{s+1 / p-2}^{p}(\Omega), \\
\operatorname{Tr} u & =g \in B_{s}^{p, p}(\partial \Omega), \quad u \in L_{s+1 / p}^{p}(\Omega),
\end{aligned}
$$

has at least one solution.

On the other hand, if $q:=(1-1 / p)^{-1}$ and $0 \leq V \in L^{r}(M)$ with $r>n$, $V>0$ on a set of positive measure in $\Omega$, then the Poisson problem with Neumann boundary condition

$$
\begin{aligned}
L u-N(x, u) & =f \in L_{1 / q-1-s, 0}^{q}(\Omega), \\
\partial_{\nu} u & =g \in B_{-s}^{q, q}(\partial \Omega), \quad u \in L_{1-s+1 / q}^{q}(\Omega),
\end{aligned}
$$

has at least one solution.

Similar results hold in dimension $n=2$ granted that (2.10) is used in lieu of (2.2) and $q_{1}, q_{2}>\left(1-\left|\frac{1}{2 p}-\frac{s}{2}\right|\right)^{-1}$ in the case of Dirichlet boundary conditions.

Finally, when $\partial \Omega \in C^{1}$, one can simply take $1<p<\infty$ and $0<s<1$ in place of (2.2), (2.10).

Proof: We shall only deal with the case of (5.2), since the case of Neumann boundary conditions is similar. To this effect, consider first the case when $s p<n-1$, and define the map $T: L^{n p /(n-1-s p)}(\Omega) \rightarrow$ $L^{n p /(n-1-s p)}(\Omega)$ by taking $v:=T u$ to be the unique solution of

$$
\begin{aligned}
L v & =f+N(x, u) \text { in } \Omega, \\
\operatorname{Tr} v & =g \in B_{s}^{p, p}(\partial \Omega), \quad v \in L_{s+1 / p}^{p}(\Omega),
\end{aligned}
$$

for each $u \in L^{n p /(n-1-s p)}(\Omega)$. Since by (5.1) and (the proof of) Lemma 2.2, $N(x, u(x))$ belongs to $L_{s+1 / p-2}^{p}(\Omega)$, Theorem 2.1 gives us that $T$ is well defined. Furthermore, there exists $C>0$ such that

$$
\begin{aligned}
& \|T u\|_{L^{n p /(n-1-s p)}(\Omega)} \\
& \quad \leq C\left(\|f\|_{L_{s+1 / p-2}^{p}}(\Omega)+\|N(x, u)\|_{L_{s+1 / p-2}^{p}(\Omega)}+\|g\|_{B_{s}^{p, p}(\partial \Omega)}\right) .
\end{aligned}
$$

The estimate (5.1) also gives us

$$
\|N(x, u(x))\|_{L_{s+1 / p-2}^{p}(\Omega)} \leq K\left(1+\|u\|_{L^{n p /(n-1-s p)}(\Omega)}^{\delta}\right)
$$


for some $K=K\left(k_{1}, k_{2}\right)>0$ independent of $u$. In particular, if we take $R>0$ big enough such that

$$
C\left(\|f\|_{L_{s+1 / p-2}^{p}(\Omega)}+\|g\|_{B_{s}^{p, p}(\partial \Omega)}+K\left(1+R^{\delta}\right)\right) \leq R,
$$

then $T$ maps the ball $\left\{h \in L^{n p /(n-1-s p)}(\Omega) ;\|h\|_{L^{n p /(n-1-s p)}(\Omega)} \leq R\right\}$ into itself.

Next, the fact that $T$ is continuous and compact is seen essentially as in Theorem 3.1. Hence, by Schauder's fixed-point theorem, the map $T$ has a fixed point $T u=u$. Since $T u$ solves (5.4) we also have that $u=T u \in L_{s+1 / p}^{p}(\Omega)$ and $\operatorname{Tr} u=\operatorname{Tr}(T u)=g$. This concludes the proof of the case $s p<n-1$.

The remaining cases discussed in the statement of the theorem are dealt with similarly and are somewhat easier; we omit the straightforward details.

Example 5.1. Our first example illustrating Theorems 3.1, 4.1 is the boundary problem

$$
(D P)_{ \pm}\left\{\begin{array}{l}
\Delta u \pm|u|^{q} u=f \in L_{s+\frac{1}{p}-2}^{p}(\Omega), \\
\operatorname{Tr} u=g \in B_{s}^{p, p}(\partial \Omega), \\
u \in L_{s+\frac{1}{p}}^{p}(\Omega) .
\end{array}\right.
$$

Theorem 3.1 gives that for the choice of the negative sign (5.8) is solvable for all $p, s$ satisfying (2.2) and $q$ such that

$$
0 \leq q<\frac{n p}{n-1-s p} \cdot \min \left\{\frac{2}{n}, 1-\frac{n-1-s p}{n p}\right\} \text { if } s p<n-1,
$$

and $q>0$ if $s p \geq n-1$, granted that $n \geq 4$. Explicit conditions, modeled upon (3.8)-(3.10), can be also given for $n=2,3$.

A case which is not directly amenable to the analysis we have developed so far corresponds to the choice of a positive sign in (5.8). In this situation, a partial answer can be obtained by relying on Theorem 2.1 and proceeding much as in [32, Chapter 6] (parenthetically, it should be pointed out that this approach works only for small data).

Finally, the range $-1<q<0$ is covered by Theorem 4.1 for either choice of the sign. Furthermore, for $(D P)_{-}$one can also establish uniqueness in this range. This can be proved by adapting the argument used in [8, Example 4.5].

Next we discuss a two dimensional curvature equation (cf. also [7], [8] for a different context). 
Example 5.2. Let $\Omega \subset M$ be a connected Lipschitz domain on a two dimensional compact manifold $M$, equipped with a Riemannian metric $g$, whose Gauss curvature is $k(x)$. The problem to be addressed is that of conformally altering $g$ to a new metric $\tilde{g}$ in $\Omega$ with a prescribed Gaussian curvature $\tilde{k}(x) \leq 0$ on $\Omega$, and such that $\left.\tilde{g}\right|_{\partial \Omega}=\left.g\right|_{\partial \Omega}$.

A well known formula, whose proof can be found in, e.g., [36, Appendix C], states that if $g$ and $\tilde{g}$ are conformally related, i.e.,

$$
\tilde{g}=e^{2 u} g,
$$

then the curvatures $\tilde{k}$ and $k$ satisfy

$$
\tilde{k}(x)=e^{-2 u}(-\Delta u+k(x)),
$$

where $\Delta=\Delta_{g}$ is the Laplace-Beltrami operator associated with the original metric $g$. Thus, in view of (5.10)-(5.11), matters come down to solving the nonlinear PDE

$$
\Delta u=k(x)-\tilde{k}(x) e^{2 u},\left.\quad u\right|_{\partial \Omega}=0 .
$$

Next we study conditions guaranteeing that the nonlinear Dirichlet problem just formulated satisfies the assumptions of Theorem 3.2. First, observe that we can rewrite (5.12) as

$$
\Delta u-\left(-\tilde{k}(x) \frac{e^{2 u}-1}{u}\right) u=k(x)-\tilde{k}(x),
$$

which, in the notation employed in Theorem 3.2, translates into

$$
a(x, u):=-\tilde{k}(x) \frac{e^{2 u}-1}{u}, \quad \text { and } \quad f(x):=k(x)-\tilde{k}(x) .
$$

Clearly, $a(x, u) \geq 0$ since, by assumption, $\tilde{k}(x) \leq 0$. Also, both (3.39) and (3.40) hold provided $\tilde{k} \in L^{r}(\Omega)$ for some $r>\left(1+\frac{1}{2 p}-\frac{s}{2}\right)^{-1}$. Hence, if we assume that the original metric tensor $g$ satisfies (1.1), as well as,

$$
g \in L_{s+\frac{1}{p}}^{p}(\Omega),
$$

then $k \in L_{s+\frac{1}{p}-2}^{p}(\Omega)$, since it has the same smoothness as $\nabla^{2} g$. Thus, granted these conditions, the existence part of Theorem 3.2 is applicable. In fact, the uniqueness condition in Theorem 3.2 also holds, since $b(x, u):=-\tilde{k}(x)\left(e^{2 u}-1\right)$, and therefore

$$
\frac{\partial}{\partial u} b(x, u)=-2 \tilde{k}(x) e^{2 u} \geq 0 .
$$


The conclusion is that for any $s, p$ satisfying (2.10), and $s p \geq 1$, we can uniquely extend $g$ satisfying (5.15) and (1.1) conformally inside $\Omega$ to a new metric $\tilde{g}$ with a prescribed curvature $\tilde{k} \leq 0, \tilde{k} \in L^{r}(\Omega)$, $r>\left(1+\frac{1}{2 p}-\frac{s}{2}\right)^{-1}$.

A discussion of the case when $k, \tilde{k}$ are locally bounded in $\mathbb{R}^{2}$ and entire solutions (with prescribed asymptotic behavior at $\infty$ ) are sought, is contained in $[\mathbf{2 2}]$.

\section{Other types of estimates and function spaces}

In this section we consider the regularity of the solution of Poisson type problems in terms of the so called nontangential maximal operator. Recall that for a function $u \in L_{\text {loc }}^{\infty}(\Omega)$, the latter is defined by

$$
u^{*}(x):=\sup _{y \in \gamma(x)}|u(x)|, \quad \text { for each } x \in \partial \Omega .
$$

Here $\gamma(x)$ is the nontangential approach region with vertex at the boundary point $x$; cf. $[\mathbf{2 7}],[\mathbf{7}]$. In what follows, we make the assumption that $\cup_{x \in \partial \Omega} \gamma(x)=\Omega$.

An issue which arises naturally in this context is that of providing an intrinsic description of the space

$$
\left\{\Delta u ; u^{*} \in L^{p}(\partial \Omega)\right\} .
$$

While at the present time this question remains open, our next definition identifies a rich linear subspace of (6.2).

Specifically, let $\Omega$ be a Lipschitz subdomain of the Riemannian manifold $M$ and fix $n / 2<\rho \leq \infty, 1 \leq q \leq \infty$. We set

$$
\begin{aligned}
\mathcal{L}_{\rho}^{q}(\Omega):=\left\{f=\sum_{j} u_{j} v_{j} ; u_{j}^{*} \in L^{q}(\partial \Omega), v_{j} \in L^{\rho}(\Omega),\right. \\
\left.\sum_{j}\left\|u_{j}^{*}\right\|_{L^{q}(\partial \Omega)}\left\|v_{j}\right\|_{L^{\rho}(\Omega)}<\infty\right\}
\end{aligned}
$$

and equip it with the norm

$$
\begin{aligned}
\|f\|_{\mathcal{L}_{\rho}^{p}(\Omega)} & :=\|f\|_{L^{1}(\Omega)} \\
& +\inf \left\{\sum_{j}\left\|u_{j}^{*}\right\|_{L^{q}(\partial \Omega)}\left\|v_{j}\right\|_{L^{\rho}(\Omega)} ; f=\sum_{j} u_{j} v_{j} \text { a.e. on } \Omega\right\} .
\end{aligned}
$$


It is helpful to observe that

$$
q \geq \rho(n-1) /[(\rho-1) n] \Longrightarrow\|u v\|_{L^{1}(\Omega)} \leq C\left\|u^{*}\right\|_{L^{q}(\partial \Omega)}\|v\|_{L^{\rho}(\Omega)} .
$$

In turn, this follows from Hölder's inequality and the estimate contained in the lemma below (cf. also [8, Lemma 4.1]).

Lemma 6.1. For every function $u$ defined in the Lipschitz domain $\Omega$ and $p>0$, there holds

$$
\|u\|_{L^{p n /(n-1)}(\Omega)} \leq C(\Omega, p)\left\|u^{*}\right\|_{L^{p}(\partial \Omega)} .
$$

Proof: The departure point is the observation that for each $\lambda>0$,

$$
\operatorname{Vol}(\{|u|>\lambda\}) \leq C \sigma\left(\left\{u^{*}>\lambda\right\}\right)^{n /(n-1)},
$$

where $d \mathrm{Vol}, d \sigma$ are, respectively, the volume element on $M$ and the surface measure on $\partial \Omega$. Indeed, if $n=2$ then this follows from definitions and simple geometrical considerations. For $n \geq 3$ the argument is slightly more involved and requires working with "tent" regions

$$
T(\mathcal{O}):=\Omega \backslash\left[\bigcup_{x \in \partial \Omega \backslash \mathcal{O}} \gamma(x)\right]
$$

associated with arbitrary open subsets $\mathcal{O}$ of $\partial \Omega$. The idea is that (6.6) is going to be a consequence of the simple inclusion

$$
\{|u|>\lambda\} \subseteq T\left(\left\{u^{*}>\lambda\right\}\right),
$$

used in concert with a general fact, to the effect that

$$
\operatorname{Vol}(T(\mathcal{O})) \leq C \sigma(\mathcal{O})^{n /(n-1)}, \quad \forall \mathcal{O} \text { open subset of } \partial \Omega .
$$

In turn, (6.10) is seen by decomposing $\mathcal{O}$ into a disjoint union of Whitney cubes $\left\{Q_{k}\right\}_{k}$ (considering $\partial \Omega$ as a space of homogeneous type), so that $T(\mathcal{O}) \subset \cup_{k} T\left(c Q_{k}\right)$ for some constant $c=c(\partial \Omega)>0$, and then writing

$$
\begin{aligned}
\operatorname{Vol}(T(\mathcal{O})) & \leq \sum_{k} \operatorname{Vol}\left(T\left(c Q_{k}\right)\right) \leq C \sum_{k} \sigma\left(Q_{k}\right)^{n /(n-1)} \\
& \leq C\left[\sum_{k} \sigma\left(Q_{k}\right)\right]^{n /(n-1)}=C \sigma(\mathcal{O})^{n /(n-1)} .
\end{aligned}
$$


Having dealt with (6.7), recall from Chebysheff's inequality that

$$
\lambda^{p} \sigma\left(\left\{u^{*}>\lambda\right\}\right) \leq\left\|u^{*}\right\|_{L^{p}(\partial \Omega)}^{p} .
$$

Consequently,

$$
\lambda^{p n /(n-1)-1} \operatorname{Vol}(\{|u|>\lambda\}) \leq C\left\|u^{*}\right\|_{L^{p}(\partial \Omega)}^{p /(n-1)} \lambda^{p-1} \sigma\left(\left\{u^{*}>\lambda\right\}\right) .
$$

Integrating both sides in $\lambda \in(0, \infty)$ readily yields $(6.6)$.

As a consequence of (6.5), $\|f\|_{L^{1}(\Omega)}$ may be omitted from (6.4) for $q$ large. It is not difficult to check that (6.4) is, indeed, a norm. Moreover, when endowed with $(6.4), \mathcal{L}_{\rho}^{q}(\Omega)$ becomes a Banach space, a claim which is straightforward to check. Our final introductory remark is that, for $n / 2<\rho \leq \infty, 1 \leq q \leq \infty$,

$$
\mathcal{L}_{\rho}^{q}(\Omega) \hookrightarrow L^{q^{*}}(\Omega), \quad \text { if } \quad \frac{1}{q^{*}} \geq \frac{1}{\rho}+\frac{n-1}{n q} .
$$

Indeed, this readily follows from (6.6), Hölder's inequality and definitions.

The next proposition is a consequence of [8, Theorem 2.9]. To state it, denote by tilde the extension by zero outside $\Omega$.

Proposition 6.2. Let $L=\Delta-V$ where $V \geq 0, V \in L^{r}(M)$ for some $r>n$ and let $L^{-1}$ be the formal inverse of $L$ on $M$. Let $1 \leq q \leq \infty$ and $\frac{n}{2}<\rho \leq \infty$. If also

$$
\frac{1}{t}>\frac{1}{q}+\frac{1}{n-1}\left(\frac{n}{\rho}-2\right),
$$

then $u:=\left.L^{-1}(\widetilde{f})\right|_{\Omega} \in C_{\text {loc }}^{0}(\Omega)$ satisfies

$$
\left\|u^{*}\right\|_{L^{t}(\partial \Omega)} \leq C\|f\|_{\mathcal{L}_{\rho}^{q}(\Omega)}
$$

for some constant $C=C(t, q, \rho)>0$. If, on the other hand,

$$
\frac{1}{t}>\frac{1}{q}+\frac{1}{n-1}\left(\frac{n}{\rho}-1\right), \quad \rho>n,
$$

then $u:=\left.L^{-1}(\tilde{f})\right|_{\Omega} \in C_{\text {loc }}^{1}(\Omega)$ obeys

$$
\left\|(\nabla u)^{*}\right\|_{L^{t}(\partial \Omega)} \leq C\|f\|_{\mathcal{L}_{\rho}^{q}(\Omega)}
$$

for some constant $C=C(t, q, \rho)>0$.

We are now prepared to discuss 
Theorem 6.3. Retain the same assumptions on $\Omega, V, s$ and $p$ as in Theorem 2.1. Assume, in addition, that $f \in \mathcal{L}_{\rho}^{q}(\Omega)$, where

$$
1 \leq q \leq \infty, \quad \frac{n}{2}<\rho \leq \infty, \quad \frac{1}{p}>\frac{1}{q}+\frac{1}{n-1}\left(\frac{n}{\rho}-2+s\right) .
$$

Then the Dirichlet Poisson problem

$$
(D P)\left\{\begin{array}{l}
L u=f \in \mathcal{L}_{\rho}^{q}(\Omega), \\
\operatorname{Tr} u=g \in B_{s}^{p, p}(\partial \Omega), \\
u \in L_{s+\frac{1}{p}}^{p}(\Omega),
\end{array}\right.
$$

has a unique solution $u \in C_{\mathrm{loc}}^{0}(\Omega)$ which also satisfies $u^{*} \in L^{t}(\partial \Omega)$, where $1 / t:=1 / p-s /(n-1)$ if $s p<n-1, t:=\infty$ if $s p>n-1$, and $t \in(1, \infty)$ is arbitrary for $s p=n-1$.

Moreover, there exists a constant $C=C(p, s, t, \Omega)>0$, independent of $V$, such that

$$
\left\|u^{*}\right\|_{L^{t}(\partial \Omega)} \leq C\left(\|f\|_{\mathcal{L}_{\rho}^{q}(\Omega)}+\|g\|_{B_{s}^{p, p}(\partial \Omega)}\right) .
$$

Finally, if $s p=n-1$, there exists $C=C(s, p, \Omega)>0$ such that for any $t \in[2, \infty)$ :

$$
\left\|u^{*}\right\|_{L^{t}(\partial \Omega)} \leq C t^{1-1 / p}\left(\|f\|_{\mathcal{L}_{\rho}^{q}(\Omega)}+\|g\|_{B_{s}^{p, p}(\partial \Omega)}\right) .
$$

Proof: Since, thanks to (6.14) and our current assumptions, $\mathcal{L}_{\rho}^{q}(\Omega) \subset$ $L_{s+1 / p-2}^{p}(\Omega)$, the solvability of (6.20) follows from Theorem 2.1. Consider first the situation $V=0$ on $\Omega$. In this case, relying on Proposition 6.2 , we see that $u$ can be written as

$$
u=\left.L^{-1}(\tilde{f})\right|_{\Omega}+\mathcal{D} h,
$$

where $\mathcal{D}$ is the double layer potential (cf. [30]) and $h \in B_{s}^{p, p}(\partial \Omega$ ) is suitably chosen. Now, $B_{s}^{p, p}(\partial \Omega) \subset L^{t}(\partial \Omega)$, if $t$ is as in the statement of our theorem. Also, the double layer potential satisfies $\left\|(\mathcal{D} f)^{*}\right\|_{L^{t}(\partial \Omega)} \leq$ $C\|f\|_{L^{t}(\partial \Omega)}$; cf. [27]. Thus, the estimate (6.21) follows. The case $s p=$ $n-1$ follows again from Lemma 2.4.

Now the claim for arbitrary $V \geq 0$ follows from the previous result for $V=0$. This is due to the fact that we once again have $0 \leq u_{V}^{( \pm)} \leq u_{0}^{( \pm)}$, i.e. the positive (and the negative) part of the solution (defined by (3.53)) for $V=0$ dominates the positive (and the negative) part of the solution for arbitrary $V \geq 0$. 
As a corollary, we can further augment the statement of Theorem 3.1 in the following way. To keep the matters simple we will consider only the case $s p<n-1$.

Theorem 6.4. Suppose that $a: \Omega \times \mathbb{R} \rightarrow \mathbb{R}$ is a Carathéodory function such that

$$
0 \leq a(x, u) \leq k_{1}(x)+k_{2}(x)|u|^{m},
$$

where $0 \leq k_{j} \in L^{q_{j}}(\Omega)$ with $q_{j}>n / 2, j=1,2$. For $1<p<\infty$, $0<s<1$, consider the following semilinear Poisson problem with Dirichlet boundary condition:

$$
\begin{aligned}
\Delta u-a(x, u) u & =f \in \mathcal{L}_{\rho}^{q}(\Omega), \\
\operatorname{Tr} u & =g \in B_{s}^{p, p}(\partial \Omega), \quad u \in L_{s+1 / p}^{p}(\Omega),
\end{aligned}
$$

where

(6.26) $1 \leq q \leq \infty, \quad \frac{n}{2}<\rho \leq \infty, \quad \frac{1}{p}>\frac{1}{q}+\frac{1}{n-1}\left(\frac{n}{\rho}-2+s\right)$.

Then there exists $\varepsilon=\varepsilon\left(\Omega, q_{1}, q_{2}\right)>0$ for which the following is true. If the pair $(p, s)$ satisfies at least one of the conditions in $(2.2), s p<n-1$ and if

$$
0 \leq m<\frac{p\left(2 q_{2}-n\right)}{q_{2}(n-1-s p)},
$$

then the boundary problem (6.25) has at least one solution $u$ which satisfies

$$
\left\|u^{*}\right\|_{L^{t}(\partial \Omega)} \leq C\left(\|f\|_{\mathcal{L}_{\rho}^{q}(\Omega)}+\|g\|_{B_{s}^{p, p}(\partial \Omega)}\right)
$$

for some $C=C(\partial \Omega, s, p, q, \rho, a)>0$ and $1 / t:=1 / p-s /(n-1)$.

Similar results are valid in dimension $n=2$ provided (2.10) is used in place of (2.2), $q_{1}, q_{2}>\left(1-\frac{1}{2 p}+\frac{s}{2}\right)^{-1}$ and $m$ satisfies (3.10). Finally, $1<p<\infty, 0<s<1$, will do in all dimensions when $\partial \Omega \in C^{1}$ as long as both $q_{1}$ and $q_{2}$ satisfy (3.3).

Proof: By virtue of (6.14) and (6.19) we have $\mathcal{L}_{\rho}^{q}(\Omega) \subset L_{s+1 / p-2}^{p}(\Omega)$. It is then natural to try to show the existence of a solution $u$ for $(6.25)$ by suitably modifying the proof of Theorem 3.1. A brief outline of the main steps is as follows. First, (6.27) ensures that the conclusion in (3.13) holds for some $r>n / 2$. This guarantees that Theorem 2.1 applies and, hence, the operator (3.11) is meaningful. Second, the key estimate (3.14) is deduced in the present setting (when $f$ is a genuine function and not just a distribution) from (6.21) (which, recall, holds 
with $C>0$ independent of $V$ for all admissible $s$ and $p$ ) and (6.6). In turn, this amounts to having (3.14) with $\theta=0$. Hence (3.18) is automatically satisfied. The role of this latter estimate is to ensure the existence of some $R>0$ for which (3.17) holds. Then, as in the proof of Theorem 3.1, using Schauder's fixed-point theorem eventually yields $u \in L^{n p /(n-1-s p)}(\Omega)$ which solves $(6.25)$.

It remains to establish (6.28). To this end, if we set $V(x):=a(x, u(x))$, we have that $V \in L^{r}(\Omega)$ where $r$ satisfies (2.3). Also, $u$ solves the linear PDE

$$
\begin{aligned}
(\Delta-V) u & =f \in \mathcal{L}_{\rho}^{q}(\Omega), \\
\operatorname{Tr} u & =g \in B_{s}^{p, p}(\partial \Omega), \quad u \in L_{s+1 / p}^{p}(\Omega) .
\end{aligned}
$$

Now, Theorem 6.3 gives that the solution of the boundary problem (6.29) is unique and the estimate (6.21) holds. It follows, that $u^{*} \in L^{t}(\partial \Omega)$, as desired.

In our last result we explore the possibility of including nonlinearities in the boundary conditions, while retaining nontangential maximal function estimates for the solution.

Theorem 6.5. Assume that $\Omega \subset M$ is an arbitrary Lipschitz domain, and assume that $a(x, u) \in L^{\infty}(\Omega \times \mathbb{R})$ is a nonnegative function, while $B(x, u)$ is a Carathéodory function on $\partial \Omega \times \mathbb{R}$ satisfying

$$
|B(x, u)| \leq k_{1}(x)+k_{2}(x)|u|^{\delta},
$$

where $k_{j} \geq 0, k_{j} \in L^{q_{j}}(\partial \Omega), j=1,2$, and

$$
\delta>\frac{1}{p}-\frac{1}{q_{2}} \geq 0, \quad q_{1} \geq p, \quad \frac{1}{p}-\frac{1}{q_{2}}>\delta\left(\frac{1}{p}-\frac{1}{n-1}\right) .
$$

Then there exists $\varepsilon=\varepsilon(\Omega)>0$ such that for each $p \in(1,2+\varepsilon)$, the semilinear Poisson problem with nonlinear Neumann boundary conditions

$$
\left\{\begin{array}{l}
\Delta u-a(x, u) u=f \in \mathcal{L}_{\rho}^{q}(\Omega), \\
(\nabla u)^{*} \in L^{p}(\partial \Omega), \\
\partial_{\nu} u+B(x, u)=g \in L^{p}(\partial \Omega),
\end{array}\right.
$$

has at least one solution, granted that

$$
\frac{1}{p}>\frac{1}{q}+\frac{1}{n-1}\left(\frac{n}{\rho}-1\right), \quad \rho>n,
$$


and either $\delta<1$, or the norms of $k_{1}, k_{2}$ are suitably small. More specifically, in the case when $\delta \geq 1$, there exist $K=K(\delta)>0$ and $M=M\left(\delta, k_{1}, k_{2}\right)>0$ such that

$$
\begin{array}{r}
\left\|k_{1}\right\|_{L^{q_{1}}(\Omega)}^{\delta-1}\left\|k_{2}\right\|_{L^{q_{2}}(\Omega)}<K \Longrightarrow \text { the problem }(6.32) \text { has at least } \\
\text { one solution, } \forall f, g \text { with }\|f\|_{\mathcal{L}_{\rho}^{q}(\Omega)}+\|g\|_{L^{p}(\partial \Omega)} \leq M .
\end{array}
$$

Once again, if $\partial \Omega \in C^{1}$, in the whole theorem we can take $1<p<\infty$. Proof: For any $R>0$, define

$$
\mathcal{O}_{R}:=\left\{u \in C_{\mathrm{loc}}^{1}(\Omega) ;\left\|(\nabla u)^{*}\right\|_{L^{p}(\partial \Omega)} \leq R\right\}
$$

and for each $u \in \mathcal{O}_{R}$ set $v:=T u$, the unique solution of the linear Neumann problem

$$
\left\{\begin{array}{l}
\Delta v-a(x, u) v=f \in \mathcal{L}_{\rho}^{q}(\Omega), \\
(\nabla v)^{*} \in L^{p}(\partial \Omega), \\
\partial_{\nu} v=g-B(x, u) \in L^{p}(\partial \Omega) .
\end{array}\right.
$$

The well posedness of the above boundary value problem follows from our assumptions, the second part of Proposition 6.2 and the results in [31]. Moreover, $v$ obeys

$$
\begin{aligned}
& \left\|(\nabla v)^{*}\right\|_{L^{p}(\partial \Omega)} \\
& \quad \leq C\left(\|f\|_{\mathcal{L}_{\rho}^{q}(\Omega)}+\|g\|_{L^{p}(\partial \Omega)}+\left\|k_{1}\right\|_{L^{q_{1}}(\Omega)}+\left\|k_{2}\right\|_{L^{q_{2}(\Omega)}} R^{\delta}\right) .
\end{aligned}
$$

Hence if $\delta<1$, we have $v \in \mathcal{O}_{R}$, provided we pick $R$ large enough so that the right side of (6.37) is $\leq R$. On the other hand, if $\delta \geq 1$ and the number $\left\|k_{1}\right\|_{L^{q_{1}(\Omega)}(\delta)}^{\delta-1}\left\|k_{2}\right\|_{L^{q_{2}(\Omega)}}$ is small, it is a simple calculus exercise to check that there exists $R>0$ so that

$$
R>C\left(\left\|k_{1}\right\|_{L^{q_{1}(\Omega)}}+\left\|k_{2}\right\|_{L^{q_{2}(\Omega)}} R^{\delta}\right) .
$$

For such $R$, if $f$ and $g$ are small in the norm, clearly $R$ is bigger than the right side of (6.37) so that, again, $v \in \mathcal{O}_{R}$. Thus, in either case there exists a choice of $R$ which guarantees that $T: \mathcal{O}_{R} \rightarrow \mathcal{O}_{R}$ is well-defined.

Finally, thanks to our hypotheses $(6.30),(6.31)$, it is not too hard to see that in fact the operator $T$ is also continuous and compact (cf. also [7, Theorem 2.4] for the latter claim). Consequently, by Schauder's theorem, $T$ has a fixed point in $\mathcal{O}_{R}$, as desired. 


\section{References}

[1] T. Aubin, "Some nonlinear problems in Riemannian geometry", Springer Monographs in Mathematics, Springer-Verlag, Berlin, 1998.

[2] C. Bennett and R. Sharpley, "Interpolation of operators", Pure and Applied Mathematics 129, Academic Press, Inc., Boston, MA, 1988.

[3] J. Bergh AND J. LÖFström, "Interpolation spaces. An introduction", Grundlehren der Mathematischen Wissenschaften 223, Springer-Verlag, Berlin-New York, 1976.

[4] L. A. Caffarelli AND X. CaBré, "Fully nonlinear elliptic equations", American Mathematical Society Colloquium Publications 43, American Mathematical Society, Providence, RI, 1995.

[5] Z.-Q. Chen, R. J. Williams and Z. ZhaO, On the existence of positive solutions for semilinear elliptic equations with singular lower order coefficients and Dirichlet boundary conditions, Math. Ann. 315(4) (1999), 735-769.

[6] B. E. Dahlberg And C. E. Kenig, Hardy spaces and the Neumann problem in $L^{p}$ for Laplace's equation in Lipschitz domains, Ann. of Math. (2) 125(3) (1987), 437-465.

[7] M. Dindoš, Existence and uniqueness for a semilinear elliptic problem on Lipschitz domains in Riemannian manifolds, Comm. Partial Differential Equations (to appear).

[8] M. Dindoš, Existence and uniqueness for a semilinear elliptic problem on Lipschitz domains in Riemannian manifolds. II, Preprint (2000).

[9] E. Fabes, O. Mendez And M. Mitrea, Boundary layers on Sobolev-Besov spaces and Poisson's equation for the Laplacian in Lipschitz domains, J. Funct. Anal. 159(2) (1998), 323-368.

[10] S. FuČÍK, "Solvability of nonlinear equations and boundary value problems", Mathematics and its Applications 4, D. Reidel Publishing Co., Dordrecht-Boston, Mass., 1980.

[11] S. FuČÍK AND A. KuFNER, "Nonlinear differential equations", Studies in Applied Mechanics 2, Elsevier Scientific Publishing Co., Amsterdam-New York, 1980.

[12] M. Giaquinta, "Introduction to regularity theory for nonlinear elliptic systems", Lectures in Mathematics ETH Zürich, Birkhäuser Verlag, Basel, 1993. 
[13] D. Gilbarg and N. S. Trudinger, "Elliptic partial differential equations of second order", Reprint of the 1998 edition, Classics in Mathematics, Springer-Verlag, Berlin, 2001.

[14] E. Hebey, "Nonlinear analysis on manifolds: Sobolev spaces and inequalities", Courant Lecture Notes in Mathematics 5, New York University, Courant Institute of Mathematical Sciences, New York, 1999.

[15] V. Isakov and A. I. Nachman, Global uniqueness for a twodimensional semilinear elliptic inverse problem, Trans. Amer. Math. Soc. 347(9) (1995), 3375-3390.

[16] D. Jerison and C. E. Kenig, The inhomogeneous Dirichlet problem in Lipschitz domains, J. Funct. Anal. 130(1) (1995), 161-219.

[17] D. Jerison And C. E. Kenig, Unique continuation and absence of positive eigenvalues for Schrödinger operators, Ann. of Math. (2) 121(3) (1985), 463-494.

[18] Z. JIN, Solvability of Dirichlet problems for semilinear elliptic equations on certain domains, Pacific J. Math. 176(1) (1996), 117-128.

[19] F. John and L. NirenberG, On functions of bounded mean oscillation, Comm. Pure Appl. Math. 14 (1961), 415-426.

[20] J. Johnsen And T. Runst, Semi-linear boundary problems of composition type in $L_{p}$-related spaces, Comm. Partial Differential Equations 22(7-8) (1997), 1283-1324.

[21] C. E. KeniG, "Harmonic analysis techniques for second order elliptic boundary value problems", CBMS Regional Conference Series in Mathematics 83, American Mathematical Society, Providence, RI, 1994.

[22] C. E. Kenig and W.-M. Ni, On the elliptic equation $L u-k+$ $K \exp [2 u]=0$, Ann. Scuola Norm. Sup. Pisa Cl. Sci. (4) 12(2) (1985), 191-224.

[23] J.-L. Lions, "Contrôle optimal de systèmes gouvernés par des équations aux dérivées partielles", Gauthier-Villars, Paris, 1968.

[24] O. Mendez and M. Mitrea, Complex powers of the Neumann Laplacian in Lipschitz domains, Math. Nachr. 223 (2001), 77-88.

[25] D. Mitrea, Layer potentials and Hodge decompositions in two dimensional Lipschitz domains, Math. Ann. 322(1) (2002), 75-101.

[26] D. Mitrea And M. Mitrea, General second order, strongly elliptic systems in low dimensional nonsmooth manifolds, in: "Harmonic analysis and boundary value problems" (Fayetteville, AR, 2000), Contemp. Math. 277, Amer. Math. Soc., Providence, RI, 2001, pp. 61-86. 
[27] D. Mitrea, M. Mitrea and M. Taylor, Layer potentials, the Hodge Laplacian, and global boundary problems in nonsmooth Riemannian manifolds, Mem. Amer. Math. Soc. 150(713) (2001), 120 pp.

[28] M. Mitrea and M. Taylor, Boundary layer methods for Lipschitz domains in Riemannian manifolds, J. Funct. Anal. 163(2) (1999), 181-251.

[29] M. Mitrea and M. Taylor, Potential theory on Lipschitz domains in Riemannian manifolds: $L^{P}$ Hardy, and Hölder space results, Comm. Anal. Geom. 9(2) (2001), 369-421.

[30] M. Mitrea and M. TaYlor, Potential theory on Lipschitz domains in Riemannian manifolds: Sobolev-Besov space results and the Poisson problem, J. Funct. Anal. 176(1) (2000), 1-79.

[31] M. Mitrea and M. TaYlor, Potential theory on Lipschitz domains in Riemannian manifolds: Hölder continuous metric tensors, Comm. Partial Differential Equations 25(7-8) (2000), 1487-1536.

[32] T. Runst And W. Sickel, "Sobolev spaces of fractional order, Nemytskij operators, and nonlinear partial differential equations", de Gruyter Series in Nonlinear Analysis and Applications 3, Walter de Gruyter \& Co., Berlin, 1996.

[33] W. SickeL, Boundedness properties of the mapping $f \mapsto|f|^{\mu}$, $0<\mu<1$ in the framework of Besov Spaces, Preprint (2000).

[34] E. M. Stein, "Singular integrals and differentiability properties of functions", Princeton Mathematical Series 30, Princeton University Press, Princeton, N.J., 1970.

[35] R. S. Strichartz, A note on Trudinger's extension of Sobolev's inequalities, Indiana Univ. Math. J. 21 (1971/72), 841-842.

[36] M. TAYlor, "Partial differential equations. Basic theory", Texts in Applied Mathematics 23, Springer-Verlag, New York, 1996.

[37] H. Triebel, "Theory of function spaces", Monographs in Mathematics 78, Birkhäuser Verlag, Basel, 1983.

[38] H. TRIEBEL, Mapping properties of nonlinear operators generated by $\Phi(u)=|u|^{\rho}$ and by holomorphic $\Phi(u)$ in function spaces of Besov-Hardy-Sobolev type. Boundary value problems for elliptic differential equations of type $\Delta u=f(x)+\Phi(u)$, Math. Nachr. 117 (1984), 193-213.

[39] G. Verchota, Layer potentials and regularity for the Dirichlet problem for Laplace's equation in Lipschitz domains, J. Funct. Anal. 59(3) (1984), 572-611. 
Martin Dindoš:

Department of Mathematics

Cornell University

310 Malott Hall Ithaca, NY, 14853

U.S.A.

E-mail address: dindos@polygon.math.cornell.edu

Marius Mitrea:

Department of Mathematics

University of Missouri at Columbia

Columbia, MO 65211

U.S.A.

E-mail address: marius@math.missouri.edu

Rebut el 17 de setembre de 2001. 